\title{
PKS 1502+106: A NEW AND DISTANT GAMMA-RAY BLAZAR IN OUTBURST DISCOVERED BY THE FERMI LARGE AREA TELESCOPE
}

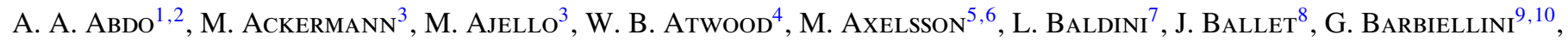

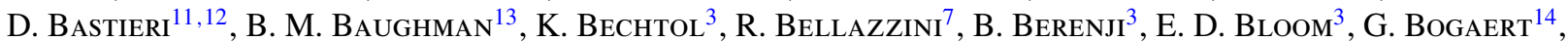

E. Bonamente ${ }^{15,16}$, A. W. Borgland ${ }^{3}$, J. Bregeon ${ }^{7}$, A. Brez ${ }^{7}$, M. Brigida ${ }^{17,18}$, P. Bruel ${ }^{14}$, T. H. Burnett ${ }^{19}$,

G. A. Caliandro ${ }^{20}$, R. A. Cameron ${ }^{3}$, P. A. Caraveo ${ }^{21}$, J. M. Casandian ${ }^{8}$, E. Cavazzuti ${ }^{22}$, C. Cecchi ${ }^{15,16}$, Ö. Çelik ${ }^{23,24,25}$, A. CheKhtman ${ }^{1,26}$, C. C. Cheung ${ }^{1,2}$, J. Chiang ${ }^{3}$, S. Ciprini ${ }^{16, *}$, R. Claus ${ }^{3}$, J. Cohen-Tanugi ${ }^{27}$, J. Conrad $^{6,28,66}$, S. Cutini $^{22}$, C. D. Dermer ${ }^{1}$, A. De Angelis ${ }^{29}$, F. De Palma ${ }^{17,18}$, S. W. Digel ${ }^{3}$, E. do Couto e Silva ${ }^{3}$, P. S. Drell ${ }^{3}$, R. Dubois ${ }^{3}$,

D. Dumora ${ }^{30,31}$, C. Farnier ${ }^{27}$, C. Favuzzi ${ }^{17,18}$, S. J. Fegan ${ }^{14}$, E. C. Ferrara ${ }^{23}$, W. B. Focke ${ }^{3}$, M. Frailis ${ }^{29}$, L. FuhrmanN ${ }^{32}$, Y. Fukazawa ${ }^{33}$, S. FunK ${ }^{3}$, P. Fusco ${ }^{17,18}$, F. Gargano ${ }^{18}$, D. GasParrini ${ }^{22}$, N. Gehrels ${ }^{23,34,35}$, S. Germani ${ }^{15,16}$, B. Giebels ${ }^{14}$, N. Giglietto ${ }^{17,18}$, F. Giordano ${ }^{17,18}$, M. Giroletti ${ }^{36}$, T. Glanzman ${ }^{3}$, G. Godfrey ${ }^{3}$, I. A. Grenier ${ }^{8}$, M.-H. Grondin ${ }^{30,31}$, J. E. Grove ${ }^{1}$, L. Guillemot ${ }^{32}$, S. Guiriec ${ }^{37}$, Y. Hanabata ${ }^{33}$, A. K. Harding ${ }^{23}$, M. HaYashida ${ }^{3}$, E. Hays ${ }^{23}$, R. E. Hughes ${ }^{13}$,

G. JóHANnesson ${ }^{3}$, A. S. JohnSON ${ }^{3}$, R. P. Johnson ${ }^{4}$, W. N. JOHNSON ${ }^{1}$, M. KADLER ${ }^{24,38,39,40}$, T. KAMAE ${ }^{3}$, H. KATAGIRI ${ }^{33}$, J. Kataoka ${ }^{41}$, M. KerR ${ }^{19}$, J. KnÖDlseder ${ }^{42}$, M. L. Kocian ${ }^{3}$, F. Kuehn ${ }^{13}$, M. Kuss ${ }^{7}$, J. Lande ${ }^{3}$, L. Latronico ${ }^{7}$,

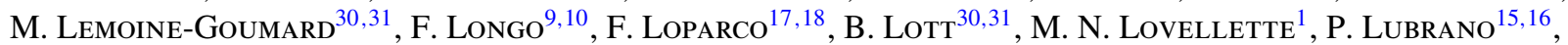

G. M. Madejski ${ }^{3}$, A. Makeev ${ }^{1,26}$, M. Marelli ${ }^{21}$, E. Massaro ${ }^{43}$, W. MaX-Moerbeck ${ }^{44}$, M. N. Mazziotta $^{18}$,

W. McConville ${ }^{23,35}$, J. E. McEnery ${ }^{23,35}$, C. Meurer ${ }^{6,28}$, P. F. Michelson ${ }^{3}$, W. Mitthumsiri ${ }^{3}$, T. Mizuno ${ }^{33}$,

A. A. Moiseev ${ }^{24,35}$, C. Monte ${ }^{17,18}$, M. E. Monzani ${ }^{3}$, A. Morselli ${ }^{45}$, I. V. Moskalenko ${ }^{3}$, S. Murgia ${ }^{3}$, P. L. Nolan ${ }^{3}$, J. P. Norris ${ }^{46}$, E. Nuss ${ }^{27}$, T. Ohsugi ${ }^{33}$, N. Omodei ${ }^{7}$, E. Orlando ${ }^{47}$, J. F. Ormes ${ }^{46}$, M. Ozaki ${ }^{48}$, D. Paneque ${ }^{3}$, J. H. Panetta ${ }^{3}$, D. Parent ${ }^{30,31}$, V. Pavlidou ${ }^{44}$, T. J. Pearson ${ }^{44}$, V. Pelassa ${ }^{27}$, M. Pepe ${ }^{15,16}$, M. Pesce-Rollins ${ }^{7}$, F. Piron ${ }^{27}$, T. A. Porter ${ }^{4}$, S. Rainò ${ }^{17,18}$, R. Rando ${ }^{11,12}$, M. Razzano ${ }^{7}$, S. Razzaque ${ }^{1,2}$, A. Readhead ${ }^{44}$, A. Reimer ${ }^{3,49}$, O. Reimer ${ }^{3,49}$, T. RePoseur ${ }^{30,31}$,

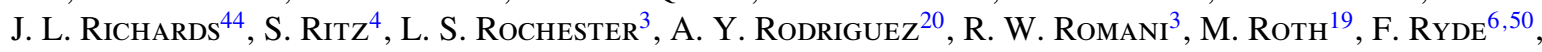

H. F.-W. Sadrozinski ${ }^{4}$, D. Sanchez ${ }^{14}$, A. Sander ${ }^{13}$, P. M. Saz Parkinson ${ }^{4}$, J. D. Scargle ${ }^{51}$, C. Sgrò ${ }^{7}$, M. S. ShaW ${ }^{3}$, E. J. Siskind ${ }^{52}$, D. A. Smith ${ }^{30,31}$, P. D. Smith ${ }^{13}$, G. Spandre ${ }^{7}$, P. Spineldi ${ }^{17,18}$, M. Stevenson ${ }^{44}$, M. S. Strickman ${ }^{1}$,

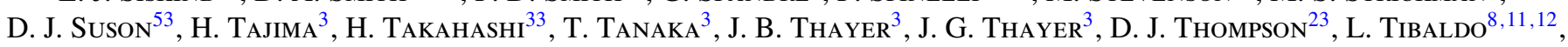
O. Tibolla ${ }^{54}$, D. F. Torres ${ }^{20,55}$, G. Tosti ${ }^{15,16}$, A. Tramacere ${ }^{3,56}$, P. Ubertini ${ }^{57}$, Y. Uchiyama ${ }^{3}$, T. L. Usher ${ }^{3}$, V. Vasileiou ${ }^{24,25}$, N. VilcheZ ${ }^{42}$, V. Vitale ${ }^{45,58}$, A. P. Waite ${ }^{3}$, P. WAnG ${ }^{3}$, B. L. WineR ${ }^{13}$, K. S. Wood ${ }^{1}$, H. Yasuda ${ }^{33}$, T. YLinen ${ }^{6,50,59}$, J. A. Zensus ${ }^{32}$, M. ZiegleR ${ }^{4}$

(The FERMI LAT COLLABORATION)

AND

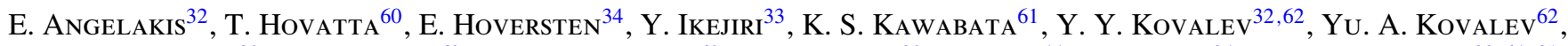
T. P. Krichbaum ${ }^{32}$, M. L. Lister ${ }^{63}$, A. Lähteenmä̈i ${ }^{60}$, N. Marchili ${ }^{32}$, P. OGLe ${ }^{44}$, C. Pagani ${ }^{34}$, A. B. PushKarev ${ }^{32,64,65}$, K. SAKimoto ${ }^{33}$, M. SASAda ${ }^{33}$, M. Tornikoski ${ }^{60}$, M. Uemura $^{61}$, M. YamanaKa ${ }^{33}$, and T. Yamashita ${ }^{61}$

${ }^{1}$ Space Science Division, Naval Research Laboratory, Washington, DC 20375, USA

${ }^{2}$ National Research Council Research Associate, National Academy of Sciences, Washington, DC 20001, USA

${ }^{3}$ W. W. Hansen Experimental Physics Laboratory, Kavli Institute for Particle Astrophysics and Cosmology, Department of Physics and SLAC National Accelerator Laboratory, Stanford University, Stanford, CA 94305, USA

${ }^{4}$ Santa Cruz Institute for Particle Physics, Department of Physics and Department of Astronomy and Astrophysics, University of California at Santa Cruz, Santa Cruz, CA 95064, USA

5 Department of Astronomy, Stockholm University, SE-106 91 Stockholm, Sweden

${ }^{6}$ The Oskar Klein Centre for Cosmoparticle Physics, AlbaNova, SE-106 91 Stockholm, Sweden

${ }^{7}$ Istituto Nazionale di Fisica Nucleare, Sezione di Pisa, I-56127 Pisa, Italy

${ }^{8}$ Laboratoire AIM, CEA-IRFU/CNRS/Université Paris Diderot, Service d'Astrophysique, CEA Saclay, 91191 Gif sur Yvette, France

${ }^{9}$ Istituto Nazionale di Fisica Nucleare, Sezione di Trieste, I-34127 Trieste, Italy ${ }^{10}$ Dipartimento di Fisica, Università di Trieste, I-34127 Trieste, Italy

${ }^{11}$ Istituto Nazionale di Fisica Nucleare, Sezione di Padova, I-35131 Padova, Italy

${ }^{12}$ Dipartimento di Fisica "G. Galilei," Università di Padova, I-35131 Padova, Italy

${ }^{13}$ Department of Physics, Center for Cosmology and Astro-Particle Physics, Ohio State University, Columbus, OH 43210, USA

${ }^{14}$ Laboratoire Leprince-Ringuet, École polytechnique, CNRS/IN2P3, Palaiseau, France

${ }^{15}$ Istituto Nazionale di Fisica Nucleare, Sezione di Perugia, I-06123 Perugia, Italy

${ }^{16}$ Dipartimento di Fisica, Università degli Studi di Perugia, I-06123 Perugia, Italy; stefano.ciprini@ @g.infn.it

${ }^{17}$ Dipartimento di Fisica "M. Merlin" dell'Università e del Politecnico di Bari, I-70126 Bari, Italy

${ }^{18}$ Istituto Nazionale di Fisica Nucleare, Sezione di Bari, 70126 Bari, Italy

${ }^{19}$ Department of Physics, University of Washington, Seattle, WA 98195-1560, USA

${ }^{20}$ Institut de Ciencies de l'Espai (IEEC-CSIC), Campus UAB, 08193 Barcelona, Spain

${ }^{21}$ INAF-Istituto di Astrofisica Spaziale e Fisica Cosmica, I-20133 Milano, Italy

22 Agenzia Spaziale Italiana (ASI) Science Data Center, I-00044 Frascati (Roma), Italy

${ }^{23}$ NASA Goddard Space Flight Center, Greenbelt, MD 20771, USA

${ }^{24}$ Center for Research and Exploration in Space Science and Technology (CRESST) and NASA Goddard Space Flight Center, Greenbelt, MD 20771, USA

${ }^{25}$ Department of Physics and Center for Space Sciences and Technology, University of Maryland Baltimore County, Baltimore, MD 21250, USA

${ }^{26}$ George Mason University, Fairfax, VA 22030, USA

${ }^{27}$ Laboratoire de Physique Théorique et Astroparticules, Université Montpellier 2, CNRS/IN2P3, Montpellier, France

${ }^{28}$ Department of Physics, Stockholm University, AlbaNova, SE-106 91 Stockholm, Sweden 
${ }^{29}$ Dipartimento di Fisica, Università di Udine and Istituto Nazionale di Fisica Nucleare, Sezione di Trieste, Gruppo Collegato di Udine, I-33100 Udine, Italy

${ }^{30}$ Université de Bordeaux, Centre d'Études Nucléaires Bordeaux Gradignan, UMR 5797, Gradignan 33175, France

${ }^{31}$ CNRS/IN2P3, Centre d'Études Nucléaires Bordeaux Gradignan, UMR 5797, Gradignan 33175, France

${ }^{32}$ Max-Planck-Institut für Radioastronomie, Auf dem Hügel 69, 53121 Bonn, Germany

${ }^{33}$ Department of Physical Sciences, Hiroshima University, Higashi-Hiroshima, Hiroshima 739-8526, Japan

${ }^{34}$ Department of Astronomy and Astrophysics, Pennsylvania State University, University Park, PA 16802, USA

${ }^{35}$ Department of Physics and Department of Astronomy, University of Maryland, College Park, MD 20742, USA

${ }^{36}$ INAF Istituto di Radioastronomia, 40129 Bologna, Italy

${ }^{37}$ Center for Space Plasma and Aeronomic Research (CSPAR), University of Alabama in Huntsville, Huntsville, AL 35899, USA

${ }^{38}$ Dr. Remeis-Sternwarte Bamberg, Sternwartstrasse 7, D-96049 Bamberg, Germany

${ }^{39}$ Erlangen Centre for Astroparticle Physics, D-91058 Erlangen, Germany

40 Universities Space Research Association (USRA), Columbia, MD 21044, USA

${ }^{41}$ Waseda University, 1-104 Totsukamachi, Shinjuku-ku, Tokyo, 169-8050, Japan

${ }^{42}$ Centre d'Étude Spatiale des Rayonnements, CNRS/UPS, BP 44346, F-30128 Toulouse Cedex 4, France

${ }^{43}$ Università di Roma "La Sapienza," I-00185 Roma, Italy

${ }^{44}$ Cahill Center for Astronomy and Astrophysics, California Institute of Technology, Pasadena, CA 91125, USA

45 Istituto Nazionale di Fisica Nucleare, Sezione di Roma "Tor Vergata," I-00133 Roma, Italy

${ }^{46}$ Department of Physics and Astronomy, University of Denver, Denver, CO 80208, USA

${ }^{47}$ Max-Planck Institut für extraterrestrische Physik, 85748 Garching, Germany

${ }^{48}$ Institute of Space and Astronautical Science, JAXA, 3-1-1 Yoshinodai, Sagamihara, Kanagawa 229-8510, Japan

${ }^{49}$ Institut für Astro- und Teilchenphysik and Institut für Theoretische Physik, Leopold-Franzens-Universität Innsbruck, A-6020 Innsbruck, Austria

${ }^{50}$ Department of Physics, Royal Institute of Technology (KTH), AlbaNova, SE-106 91 Stockholm, Sweden

${ }^{51}$ Space Sciences Division, NASA Ames Research Center, Moffett Field, CA 94035-1000, USA

${ }^{52}$ NYCB Real-Time Computing Inc., Lattingtown, NY 11560-1025, USA

${ }^{53}$ Department of Chemistry and Physics, Purdue University Calumet, Hammond, IN 46323-2094, USA

${ }^{54}$ Max-Planck-Institut für Kernphysik, D-69029 Heidelberg, Germany

${ }^{55}$ Institució Catalana de Recerca i Estudis Avançats (ICREA), Barcelona, Spain

${ }^{56}$ Consorzio Interuniversitario per la Fisica Spaziale (CIFS), I-10133 Torino, Italy

${ }^{57}$ INAF-Istituto di Astrofisica Spaziale e Fisica Cosmica, I-00133 Roma, Italy

${ }^{58}$ Dipartimento di Fisica, Università di Roma “Tor Vergata," I-00133 Roma, Italy

${ }^{59}$ School of Pure and Applied Natural Sciences, University of Kalmar, SE-391 82 Kalmar, Sweden

${ }^{60}$ Metsähovi Radio Observatory, Helsinki University of Technology TKK, FIN-02540 Kylmala, Finland

${ }^{61}$ Hiroshima Astrophysical Science Center, Hiroshima University, Higashi-Hiroshima, Hiroshima 739-8526, Japan

62 Astro Space Center of the Lebedev Physical Institute, 117810 Moscow, Russia

${ }^{63}$ Department of Physics, Purdue University, West Lafayette, IN 47907, USA

${ }^{64}$ Crimean Astrophysical Observatory, 98409 Nauchny, Crimea, Ukraine

65 Pulkovo Observatory, 196140 St. Petersburg, Russia

Received 2009 July 29; accepted 2009 December 21; published 2010 January 22

\section{ABSTRACT}

The Large Area Telescope (LAT) on board the Fermi Gamma-ray Space Telescope discovered a rapid ( $\sim 5$ days duration), high-energy $(E>100 \mathrm{MeV})$ gamma-ray outburst from a source identified with the blazar PKS 1502+106 (OR 103, S3 1502+10, $z=1.839$ ) starting on 2008 August 5 ( $\sim 23$ UTC, MJD 54683.95), and followed by bright and variable flux over the next few months. Results on the gamma-ray localization and identification, as well as spectral and temporal behavior during the first months of the Fermi all-sky survey, are reported here in conjunction with a multiwaveband characterization as a result of one of the first Fermi multifrequency campaigns. The campaign included a Swift ToO (followed up by a 16 day observation on August 7-22, MJD 54685-54700), VLBA (within the MOJAVE program), Owens Valley Radio Observatory (OVRO) $40 \mathrm{~m}$, Effelsberg-100 m, Metsähovi-14 m, RATAN-600, and Kanata-Hiroshima radio/optical observations. Results from the analysis of archival observations by INTEGRAL, XMM-Newton, and Spitzer space telescopes are reported for a more complete picture of this new gamma-ray blazar. PKS $1502+106$ is a sub-GeV peaked, powerful flat spectrum radio quasar (luminosity at $E>100 \mathrm{MeV}, L_{\gamma}$, is about $1.1 \times 10^{49} \mathrm{erg} \mathrm{s}^{-1}$, and black hole mass likely close to $10^{9} M_{\odot}$ ), exhibiting marked gamma-ray bolometric dominance, in particular during the asymmetric outburst $\left(L_{\gamma} / L_{\mathrm{opt}} \sim 100\right.$, and 5 day averaged flux $F_{E>100 \mathrm{MeV}}=2.91 \pm 1.4 \times 10^{-6} \mathrm{ph} \mathrm{cm}^{-2} \mathrm{~s}^{-1}$ ), which was characterized by a factor greater than 3 of flux increase in less than $12 \mathrm{hr}$. The outburst was observed simultaneously from optical to X-ray bands $\left(F_{0.3-10 \mathrm{keV}}=2.18_{-0.12}^{+0.15} \times 10^{-12} \mathrm{erg} \mathrm{cm}^{-2} \mathrm{~s}^{-1}\right.$, and hard photon index $\sim 1.5$, similar to past values) with a flux increase of less than 1 order of magnitude with respect to past observations, and was likely controlled by Comptonization of external-jet photons produced in the broad-line region (BLR) in the gamma-ray band. No evidence of a possible blue bump signature was observed in the optical-UV continuum spectrum, while some hints for a possible 4 day time lag with respect to the gamma-ray flare were found. Nonetheless, the properties of PKS 1502+106 and the strict optical/UV, X-, and gamma-ray cross-correlations suggest the contribution of the synchrotron self-Compton (SSC), in-jet, process should dominate from radio to X-rays. This mechanism may also be responsible for the consistent gamma-ray variability observed by the LAT on longer timescales, after the ignition of activity at these energies provided by the BLR-dissipated outburst. Modulations and subsequent minor, rapid flare events were detected, with a general fluctuation mode between pink-noise and a random-walk. The averaged gamma-ray spectrum showed a deviation from a simple power law, and can be described by a log-parabola curved model peaking around $0.4-0.5 \mathrm{GeV}$. The maximum energy of photons detected from the 
source in the first four months of LAT observations was $15.8 \mathrm{GeV}$, with no significant consequences on extragalactic background light predictions. A possible radio counterpart of the gamma-ray outburst can be assumed only if a delay of more than three months is considered on the basis of opacity effects at $\mathrm{cm}$ and longer wavelengths. The rotation of the electric vector position angle observed by VLBA from 2007 to 2008 could represent a slow field ordering and alignment with respect to the jet axis, likely a precursor feature of the ejection of a superluminal radio knot and the high-energy outburst. This observing campaign provides more insight into the connection between MeV-GeV flares and the moving, polarized structures observed by the VLBI.

Key words: galaxies: active - galaxies: jets - gamma rays: general - quasars: general - quasars: individual (PKS 1502+106) - X-rays: galaxies

Online-only material: color figures

\section{INTRODUCTION}

The Large Area Telescope (LAT), on board the Fermi Gamma-ray Space Telescope (formerly GLAST; Ritz 2007), was successfully launched by NASA on 2008 June 11 from Cape Canaveral, Florida, on a Delta II Heavy launch vehicle. While still in the commissioning and checkout phase, it discovered and monitored bright, flaring gamma-ray emission above $100 \mathrm{MeV}$ from a source identified with the blazar PKS 1502+106 (historically also known as OR 103 and S3 1502+10). The large field of view, effective area, and sensitivity, and the nominal survey observational mode make Fermi-LAT an unprecedented all-sky monitor of $\gamma$-ray flares and source variability (see, e.g., McEnery 2006; Thompson 2006; Lott et al. 2007; Atwood et al. 2009).

At the beginning of 2008 August, PKS 1502+106 was the second brightest extragalactic source in the $\gamma$-ray sky, exhibiting a sudden high-energy outburst announced in ATel \#1650. This outburst successfully triggered the first (unplanned) Fermi multifrequency campaign. Major renewed gamma-ray activity observed by Fermi in 2009 January was announced via ATel \#1905.

PKS $1502+106$ is a luminous, quasar-like (optically broadline and flat radio spectrum) active galactic nucleus (AGN) discovered during the $178 \mathrm{MHz}$ pencil beam survey from the Mullard Radio Astronomy Observatory, Cambridge, UK (appearing in a list not included in the 4C catalog; Crowther \& Clarke 1966; Williams et al. 1967), and was re-observed and characterized as an extragalactic source by both the Australian National Radio Astronomy Observatory of Parkes, NSW, Australia (Day et al. 1966; ID: PKS 1502+106), and the Ohio State University ("Big Ear") Radio Observatory, Delaware, $\mathrm{OH}$, USA (Fitch et al. 1969; ID: OR 103). The source exhibited substantial radio flux variations (factor $>2$ ), a high degree of linear polarization, a core-dominated, one-sided, and curved radio jet with a variable, a complex morphology at very long baseline interferometry (VLBI) scales (An et al. 2004; Lister et al. 2009a), and a compact large-scale structure. Eleven Very Long Baseline Array (VLBA) observations at $15.4 \mathrm{GHz}$ performed between 1997 August and 2007 August showed an FWHM major beam axis in the range 1.02-1.57 mas, a minor axis beam axis of 0.5 mas, and a total flux density in the range $0.88-1.93 \mathrm{Jy}$ (Lister et al. 2009a), and an apparent jet speed of (14.8 \pm 1.2$) c$ (Lister et al. 2009b). The 22 and $37 \mathrm{GHz}$ flux history shows several long-term flares ( $>1 \mathrm{Jy}$ variations, i.e., $\sim 60 \%$ of the total flux range span, on typical timescales of a year, and peak fluxes well above $2 \mathrm{Jy}$ ), with at least five flares and an average trend

\footnotetext{
${ }^{66}$ Royal Swedish Academy of Sciences Research Fellow, funded by a grant from the K. A. Wallenberg Foundation.

* Corresponding author: stefano.ciprini@pg.infn.it
}

that was slightly increasing from 1988 to mid-2004 (Teräsranta et al. 2005). WMAP fluxes at similar frequencies (K, Ka, and Q bands) are in agreement with these flux ranges (López-Caniego et al. 2007). The Doppler factor estimated from the observed $37 \mathrm{GHz}$ variability and brightness temperature (Hovatta et al. 2009) agrees with the jet speed (14.6c) cited above, a Doppler factor $\mathcal{D}_{\text {var }}=12$, and viewing angle $\theta_{\text {var }}=4.7$.

This radio blazar was identified in the optical band by Blake (1970) with a position refinement by Argue \& Sullivan (1980), while an initial spectroscopic inspection was performed by Burbidge \& Strittmatter (1972). Variations $>2.5$ mag were observed in its optical flux history (Palomar-Quest and Catalina Sky Surveys, ATel \#1661), together with a variable and relatively high degree (up to $20 \%$ ) of linear polarization, pointing out a dominant synchrotron emission with no observed dilution by thermal components. The redshift of PKS $1502+106$, as confirmed by the good signal-to-noise ratio $(\mathrm{S} / \mathrm{N})$ spectrum of the Sloan Digital Sky Survey (SDSS; $z=1.8385 \pm 0.0024$ at high confidence), is in agreement with the value $z=1.839$ estimated previously by Smith et al. (1977). A less remote value $(z=0.56)$ is reported in other works (Burbidge \& Strittmatter 1972; Wright et al. 1979; Wilkes et al. 1983), although the possible multiple Mg II absorption system (pointed out by a feature shortward of the $4388 \AA$ emission line) would be very unusual for a low-redshift object.

Serendipitous X-ray data of PKS $1502+106$ are available because the source lies about $7^{\prime} \mathrm{NE}$ of the bright Seyfert type1 galaxy Mkn 841, although only one multifrequency work dedicated to this blazar (George et al. 1994) has appeared previously. Early X-ray observations (ROSAT, ASCA) showed low-amplitude variations on short timescales (factor $>2$ on timescales of a year), a flat $0.1-10 \mathrm{keV}$ photon index $\Gamma_{X}$ between 1.4 and 1.9, and an intrinsic X-ray luminosity of $L_{2-10 \mathrm{keV}}=1.2 \times 10^{46} \mathrm{erg} \mathrm{s}^{-1}$, and a $2-10 \mathrm{keV}$ flux in the range 4.9-6.54 $\times 10^{-13}$ erg cm $\mathrm{cm}^{-2} \mathrm{~s}^{-1}$ (George et al. 1994; Akiyama et al. 2003; Watanabe et al. 2004). PKS 1502+106 was speculated to be a possible $\gamma$-ray source before the LAT discovery because of the superluminal motions of jet components (up to $187 \pm 15 \mu \mathrm{as} \mathrm{yr}^{-1}$; Lister \& Homan 2005; An et al. 2004), and the multiwaveband spectral indexes $\alpha_{\mathrm{rx}}$ and $\alpha_{\mathrm{ox}}$ (consistent with other FSRQs detected by EGRET; George et al. 1994). Only modest intrinsic X-ray absorption was suggested by this work, and the optical and near-IR reddening claimed in Watanabe et al. (2004) is probably due to the synchrotron jet dominance at these low frequencies rather than by absorption from inner nuclear light.

A relation involving the misalignment between the $\mathrm{pc}$ - and kpc-scale radio structure (position angle) and the $\gamma$-ray emission was postulated as well (Cooper et al. 2007). However, only a cumulative $2 \sigma$ upper limit by EGRET of $7 \times 10^{-8} \mathrm{ph} \mathrm{cm}^{-2} \mathrm{~s}^{-1}$ 
was reported (Phase/Cycle I, combined Viewing Periods: 24.0 to 25.0, i.e., 1992 April 2-23; Fichtel et al. 1994), and the source was likewise undetected in the following EGRET cycles (Hartman et al. 1999; Casandjian \& Grenier 2008).

In the following, we use a $\Lambda \mathrm{CDM}$ (concordance) cosmology with values given within $1 \sigma$ of the WMAP results (Komatsu et al. 2009), namely, $h=0.71, \Omega_{m}=0.27$, and $\Omega_{\Lambda}=0.73$, and a Hubble constant value $H_{0}=100 \mathrm{~h} \mathrm{~km} \mathrm{~s}^{-1} \mathrm{Mpc}^{-1}$.

In Section 2, first results on the $\gamma$-ray identification, the observed $\mathrm{MeV}-\mathrm{GeV}$ outburst and the subsequent four months of monitoring by the Fermi-LAT are described. In Section 3, multifrequency results obtained through simultaneous opticalUV-X-ray observations by Swift (thanks to a 16 day long monitoring following a triggered Target of Opportunity, ToO), and by radio-optical observatories (the $40 \mathrm{~m}$ dish telescope of the Owens Valley Radio Observatory (OVRO), the Effelsberg $100 \mathrm{~m}$ dish radio telescope, the ring radio telescope RATAN-600, the VLBA within the MOJAVE program, and the Kanata telescope of the Higashi-Hiroshima Observatory) are summarized. In addition, past and unpublished observations by the XMM-Newton and Spitzer space telescopes are analyzed and presented in Section 4 for a more complete picture. Finally, in Sections 5 and 6, discussion and concluding remarks are reported.

\section{GAMMA-RAY OBSERVATIONS AND RESULTS BY FERMI-LAT}

\subsection{LAT Observations}

The LAT instrument is a pair tracker-converter telescope comprising a modular array of 16 towers - each with a tracker based on silicon micro-strip detector technology-and a calorimeter based on a hodoscopic array of $96 \mathrm{CsI}(\mathrm{Tl})$ crystals, surrounded by an Anti-coincidence Detector capable of measuring the directions and energies of cosmic $\gamma$-ray photons with energies from $20 \mathrm{MeV}$ to $>300 \mathrm{GeV}$ (for details, see, e.g., Bellazzini et al. 2002; Michelson 2007; Atwood et al. 2007, 2009; Abdo et al. 2009d).

The reduction and analysis of LAT data were performed using the Science Tools v.9.8, based in particular on an unbinned maximum likelihood estimator of the spectral model parameters (gtlike tool). Events were selected using the instrument response functions (IRFs) P6_V1_DIFFUSE. This selection provides the cleanest set of events (in terms of direction, energy reconstruction, and background rejection) at the cost of reduced effective area at low energies, and takes into account the differences between front- and back-converting events. To minimize contamination by Earth albedo $\gamma$-ray events that have reconstructed directions with angles with respect to the local zenith $>105^{\circ}$ have been excluded. For this object with high Galactic latitude, events are extracted within a $10^{\circ}$ acceptance cone centered at the PKS $1502+106$ radio position. This cone, substantially larger than the $68 \%$ containment angle of the PSF at the lowest energies, provides sufficient events to accurately constrain the diffuse emission components. The gtlike model includes the PKS $1502+106$ point source component, two other point sources from the three-month catalog (both faint and low-confidence sources with $\mathrm{TS} \simeq 0.9 \%$ of the $\mathrm{TS}$ value of PKS $1502+106$ for the same period), a component for the Galactic diffuse emission (GALPROP code; see, e.g., Moskalenko et al. 2003, and references therein), and an isotropic component including the extragalactic diffuse emission and the residual background from cosmic rays.
The Fermi-LAT data of PKS 1502+106 presented here were obtained during the first four months of the LAT survey (2008 August-December). In this period PKS 1502+106 was one of the most persistently bright, variable sources in the highenergy sky and almost certainly the source with the highest luminosity. The background contribution within a few degrees was only a small fraction of the source count rate, with no nearby source confusion. The time interval was sufficient for a fine determination of the average spectrum, for a first look at the mid-timescale variability and detection of posterior flares, for a refined localization, and a first cross comparison with the other multifrequency monitoring data. The first $\gamma$-ray detection of PKS $1502+106$ by the LAT occurred in 2008 July, when it was confirmed by the high-level Automatic Science Processing pipeline monitoring (ASP; Chiang et al. 2006, 2007), based on a wavelet-based (pgwave) quick-look detection tool (e.g., Damiani et al. 1997; Marcucci et al. 2004; Ciprini et al. 2007a) and a maximum likelihood analysis, and by the LAT Source Catalog algorithm (mr_filter) based on wavelet analysis in the Poisson regime (Starck \& Pierre 1998) and the peak-finding tool sExtractor (Bertin \& Arnouts 1996). The rapid and markedly time-asymmetric $\gamma$-ray outburst announced in ATel \#1650 and triggering an unplanned ToO multifrequency campaign was seen from 2008 August 5 (about 23 UTC) until about 2008 August 11 ( $\sim 5$ days duration), with a fast rise, slower decay, and an approximately two-day sustained peak flux.

Some caveats related to the preflight IRFs (P6_V1), which overestimated the acceptance at low energies, are briefly described in Abdo et al. (2009b).

\subsection{Gamma-ray Source Localization, Association, and Identification}

The LAT PSF and sensitivity provides an unprecedented angular resolution in gamma rays $(68 \%$ containment radius better than $\sim 1^{\circ}$ at $1 \mathrm{GeV}$; Atwood et al. 2007, 2009; Cecchi et al. 2007; Abdo et al. 2009c, 2009d), making the association and identification processes less difficult than in previous experiments. In the case of this very bright $\gamma$-ray source, we obtainedbeyond the good spatial "association"-a firm "identification" with PKS 1502+106. The three-month bright source list results (Abdo et al. 2009c; ID: 0FGL J1504.4+1030), provided a good initial localization: R.A.: 226.12, decl.: $+10.51, r_{95}=0.05$, and $\sqrt{\mathrm{TS}}=88.2\left(r_{95}\right.$ being the radius of $95 \%$ confidence region and TS the likelihood test statistic from the $200 \mathrm{MeV}$ to $100 \mathrm{GeV}$ analysis). Application of the pointlike tool (Burnett 2007; Abdo et al. 2009c) on a much longer (ninemonth, 2008 August-2009 April) LAT data set with very high statistics, provided an excellent localization (outlined in Figure 1): R.A.: 226.10179, decl.: $+10.4927, \Delta=0.0027$, with $68 \%$ and $95 \%$ LAT error circles of 0.0077 and 0.0124 , respectively (statistical only). Studies of bright source localizations indicate a systematic uncertainty in the localization of $<30^{\prime \prime}$ that can be taken as an estimate of the systematics with this tool (more details on the production of pointlike density maps and localization are described in Camilo et al. 2009). The relevant improvement in the localization carried out on a nine-month baseline is due to the high variability that occurred with this source. These localization values are in agreement with the VLBI radio and optical positions of PKS 1502+106, the VLA contours and the Swift XRT error box (Figure 1). PKS 1502+106 is the only bright VLA radio source (calibrator source list) located within the LAT 95\% confidence circle. The Seyfert galaxy Mkn 841 (observed with a hard X-ray cutoff; see Section 4) is 


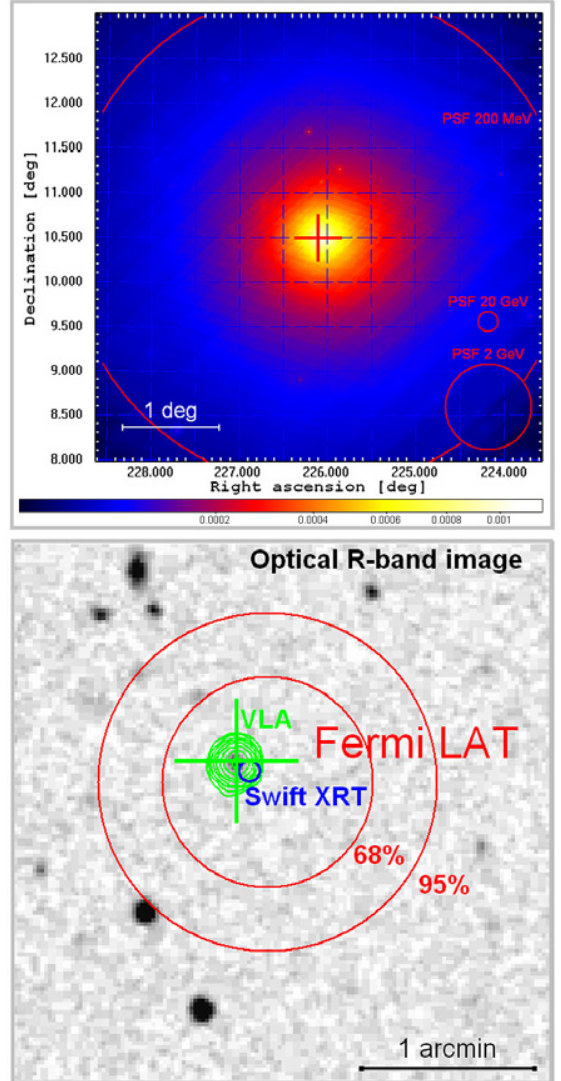

Figure 1. Top panel: LAT count map cumulated on a nine-month (2008 August2009 April) baseline, weighted and smoothed by the point-spread function (PSF) such that higher energy photons are mapped to higher intensities. The map is in arbitrary units in the energy range $0.1-100 \mathrm{GeV}$ and in a $2.5 \times 2.5$ region centered on PKS 1502+106. The qualitative circle sizes of the PSF at $200 \mathrm{MeV}, 2 \mathrm{GeV}$, and $20 \mathrm{GeV}$ are outlined for reference. Bottom panel LAT source localization with $95 \%$ and $68 \%$ uncertainty radii (red circles) superimposed on an arcmin-scale ( $R$ band) optical image showing also the X-ray counterpart error box by the Swift-XRT observations and the radio position and intensity contours by VLA of PKS $1502+106$. The best LAT source position, calculated on the same nine-month period, with the pointlike tool is R.A.: 226.10179 , decl.: $+10.4927, \Delta=0.0027$, with $68 \%$ and $95 \%$ LAT error circles of 0.0077 and 0.0124 , respectively.

(A color version of this figure is available in the online journal.)

positioned well outside of these localization circles, as are other HB89-catalog quasars in this region.

Beyond the excellent spatial association, the most secure and distinctive signature for firm identification of this new gamma-ray source found by Fermi is the observed correlation between the $\gamma$-ray, X-ray, and optical-UV variability (see Sections 3 and 4). This object was also a member of the pre-launch CGRaBS (Healey et al. 2008; object ID: CGRaBS J1504+1029) and Roma-BZCAT (Massaro et al. 2009; object ID: BZQ J1504+1029) catalogs listing candidate gamma-ray blazars. Finally, a method based on a "figure of merit" (described in Sowards-Emmerd et al. 2003, 2005) for this LAT source position provides a very high likelihood of identification with PKS 1502+106.

\subsection{Gamma-ray Temporal Behavior}

The typically bright $\gamma$-ray flux and the enduring activity shown by PKS $1502+106$ in $\gamma$-rays allowed a firm detection of the source by the LAT on a daily basis. Figure 2 shows the light curve (daily bins, $E>100 \mathrm{MeV}$ ) extracted with the gtlike tool over the first four and half months of LAT all-sky survey. A fast-rising, markedly asymmetric and bright outburst was found, with a factor $>3$ of increase in flux in less than $12 \mathrm{hr}$. The integrated flux at $E>100 \mathrm{MeV}$ averaged in the $12 \mathrm{hr}$ bin of the peak emission (between 2008 August 5 and 6, i.e., DOY 218-219; DOY: Day of Year) was $F_{E>100 \mathrm{MeV}}=(3.7 \pm 0.7) \times 10^{-6} \mathrm{ph} \mathrm{cm}^{-2} \mathrm{~s}^{-1}$ (statistical only), as measured when the LAT instrument was still in commissioning and checkout phase (all-sky nominal mode). The emission from PKS 1502+106 then faded more slowly in the following days, and the entire outburst interval spanned 2008 August 5 around 23 UTC until to 2008 August 11 around 00 UTC (i.e., DOY, 218.95-224.0, 5 days duration, Figure 2). The peak flux appeared elevated for less than two days, rivaling the brightest apparent flux of other extragalactic sources at that time (Section 2.1). The finer, $12 \mathrm{hr}$ bin light curve ( $\sim 8$ Fermi orbits, ensuring still similar exposures) reported in the right inset panel of Figure 2, shows the elevated flux held for at least 1.5 days, while the slower fading decay exhibits a high flux $\left(F_{E>100 \mathrm{MeV}}>2 \times 10^{-6} \mathrm{ph} \mathrm{cm}^{-2} \mathrm{~s}^{-1}\right)$ plateau, or a secondary superposed flare, that extended for about 2.5 days. During this outburst a "harder when brighter" spectral trend is suggested, despite the photon index error dispersion (see the daily photon indices reported on the right inset panel of Figure 2). The gtlike performances and the current IRFs used did not allow to go below a daily binning in the extrapolation of the photon index values, and this was possible in an acceptable way with respect to amplitude of the statistical error only for the high-flux and high photon count statistics available around the outburst epoch.

A consistent level of variability, with a couple of minor but relevant rapid flares, occurred after the major outburst with fluctuations on timescales of weeks. Renewed activity and increased average brightness from the end of 2008 November were observed. Two rapid flares approached a maximum peak flux of $F_{E>100 \mathrm{MeV}} \sim 2 \times 10^{-6} \mathrm{ph} \mathrm{cm}^{-2} \mathrm{~s}^{-1}$ (daily bin estimations), on September 6 (DOY 250), where a simultaneous crosscorrelated optical flare was observed as well, and on October 30 (DOY 304). Visual inspection of the light curve reported in Figure 2 suggests a period of higher activity beginning in mid 2008 November (after about DOY 320), and, in general, a series of modulations occurring on about a one-month timescale, with faster fluctuations and rapid flare episodes superposed. The power spectral density (PSD) shows a power-law dependence, $P(f) \propto 1 / f^{1.3}$. Similar timescale dependence is exhibited by the first-order structure function (Figure 3 ) and by the autocorrelation function. More detailed variability analysis for this and other blazars using a longer data set will be presented in Abdo et al. (2010a).

\subsection{Gamma-ray Spectra}

We have analyzed the time-averaged spectra of PKS $1502+106$ for three intervals: the high state of the outburst (DOY, 218.95-224.0, i.e., 2008 August 5-11, about 5 days); the post-flare period characterized by an intermediate brightness level and during which simultaneous Swift observations were performed (DOY 224.0-235.42, i.e., 2008 August 11-22, about 11.4 days); the longer and heterogeneous period that includes the outburst and the following four months of Fermi-LAT monitoring (2008 August 2 to December 7, 126 days, where the source displayed different stages of activity and significant variability). Events below $200 \mathrm{MeV}$ were excluded from these analyses because of calibration uncertainties at those energies. An isotropic background model used as PKS 1502+106 was 


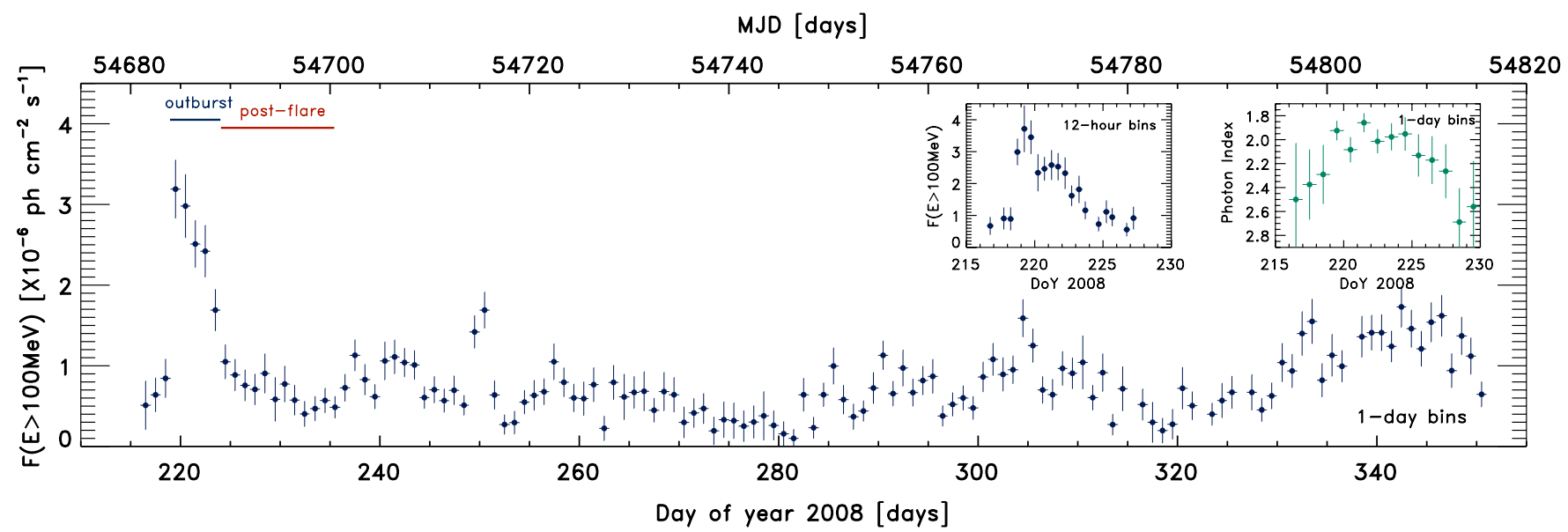

Figure 2. Main panel: likelihood flux $(E>100 \mathrm{MeV})$ light curve obtained in daily bins from 2008 August 2 to December 15. The outburst state and the subsequent post-flare (a lower and intermediate level brightness, far from the faintest state observed) period with simultaneous monitoring by Swift are represented by the two horizontal lines. Left inset plot: a zoom on the corresponding flux light curve around the outburst period obtained using finer, $12 \mathrm{hr}$ bins (lower statistics). Right inset plot: the gamma-ray $(E>100 \mathrm{MeV})$ photon index values for the same period using daily bins as in the main panel light curve.

(A color version of this figure is available in the online journal.)

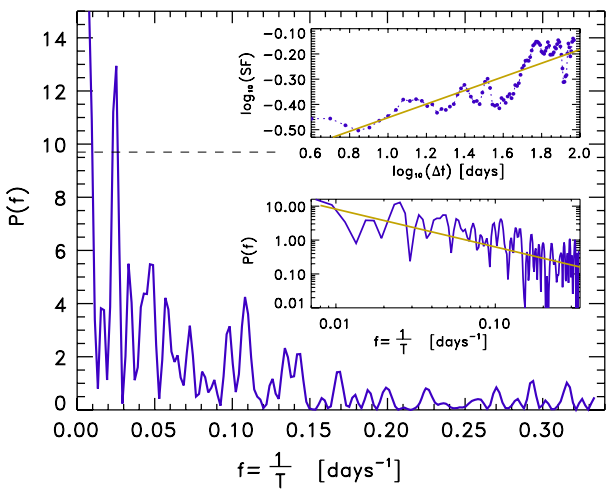

Figure 3. Time series analysis of the LAT light curve presented in Figure 2: the periodogram, first-order structure function (upper inset), and power spectrum (lower inset). These functions show a variability with a power spectrum consistent with $1 / f^{1.3}$ fluctuations. This indicates a variability mode placed between flickering and shot noise. The horizontal dashed line represents the 0.01 false alarm probability threshold ( $99 \%$ significance that the signal detection is not wrong).

(A color version of this figure is available in the online journal.)

very bright relative to other point sources during the period stated above, because it is located at high Galactic latitude, and because checks with more complex models provided no significant difference. Furthermore, no appreciable differences were observed using different acceptance cone radii for the event extraction.

The spectra for the post-flare and cumulative four-month data sets can be consistent with a log-parabola (LP) model, $d N / d E \propto E^{-(\alpha+\beta \log (E))}$ (see, e.g., Landau et al. 1986; Inoue \& Takahara 1996; Fossati et al. 2000; Massaro et al. 2004; Perlman et al. 2005). The likelihood ratio test (Mattox et al. 1996) rejects the hypothesis that the spectrum is a power law (null hypothesis) against the one that the spectrum is curved as an LP model. This model is preferred over a simple power-law model at the $11.4 \sigma$ significance level. Broken power-law (BPL) fits show a similar improvement over single power-law models, but we find no evidence to prefer BPL over the LP representation. For the full time interval characterized by very high statistics, the logarithm of the likelihood increases significantly when allowing $\beta$ to vary, and an increase of the value for the BPL with respect to simple power-law model of the same order of the increase for the
Table 1

Summary of the Unbinned Likelihood Spectral Fit Above $100 \mathrm{MeV}$

\begin{tabular}{|c|c|}
\hline Interval [MJD (DOY)] & Best-fit Model and Parameters \\
\hline $\begin{array}{l}\text { All observations } \\
54682.680(217.680) \\
54775.580(310.580)\end{array}$ & $\begin{array}{c}\text { Log-parabola } \\
\alpha=1.94 \pm 0.05 \\
\beta=0.10 \pm 0.02 \\
F_{E>100 \mathrm{MeV}}=6.90 \pm 0.34 \times 10^{-7}\left(\mathrm{ph} \mathrm{cm}^{-2} \mathrm{~s}^{-1}\right)\end{array}$ \\
\hline $\begin{array}{l}\text { Outburst/high state } \\
54683.955(218.955) \\
54688.985(223.985) \\
\end{array}$ & $\begin{array}{c}\text { Power law } \\
\Gamma=2.06 \pm 0.017 \\
F_{E>100 \mathrm{MeV}}=29.1 \pm 1.4 \times 10^{-7}\left(\mathrm{ph} \mathrm{cm}^{-2} \mathrm{~s}^{-1}\right) \\
\end{array}$ \\
\hline $\begin{array}{l}\text { Post-flare/lower state } \\
54689.063(224.063) \\
54775.580(310.580)\end{array}$ & $\begin{array}{c}\text { Log-parabola } \\
\alpha=1.87 \pm 0.20 \\
\beta=0.18 \pm 0.08 \\
F_{E>100 \mathrm{MeV}}=5.32 \pm 1.03 \times 10^{-7}\left(\mathrm{ph} \mathrm{cm}^{-2} \mathrm{~s}^{-1}\right)\end{array}$ \\
\hline
\end{tabular}

LP versus power-law test is not observed. The LP description introduces the advantage of only one extra parameter (while BPL model adds two parameters) with respect to the simple power-law model, it allows modeling of mild spectral curvatures with no abrupt cutoffs, and provides a better phenomenological physical interpretation. On the other hand, there can still be caveats when using gtlike with a BPL model, in particular in determining the break energy when statistics do not allow a high number of energy bins. It is also plausible that an energy spectrum averaged over a long period of time, and containing different activity stages with time varying hardness, may exhibit an apparent curvature. Finally, this does not exclude BPL model if the spectrum is extracted in different time intervals.

The average spectrum during the outburst state is consistent with a simple power-law model, $d N / d E \propto E^{-\Gamma}$. The outburst state shows a rather hard spectrum, suggesting a maximum peak in the $\mathrm{MeV}$ energy bands (in the $\nu F_{v}$ representation), in agreement with the LP peaks found for the spectra cited above. The extrapolated and averaged fluxes integrated above $100 \mathrm{MeV}$, and the spectral-fit parameters for all three periods are shown in Table 1.

\section{SIMULTANEOUS MULTIFREQUENCY OBSERVATIONS}

Because of the reasonably uniform exposure and high sensitivity of the LAT, and the broadband radio-to-gamma-ray emis- 
sion of this kind of AGN, simultaneous multifrequency data are very important to the investigation of the physical properties of supermassive black holes and relativistic jets, beyond the benefit of a firm source identification (Section 2.2). With this in mind, several campaigns on a few selected objects, or ToO list of candidates for flaring sources, were prepared prelaunch by the Fermi collaboration (Tosti 2007; Thompson 2007). PKS $1502+106$ was a previously unknown $\gamma$-ray source, with no pre-planned multifrequency campaign. But following the LAT outburst (reported in ATel \#1650), a ToO campaign was initiated on 2008 August 7. This was the first Fermi multifrequency campaign that had not been planned pre-launch, and saw triggers for ToO pointings by INTEGRAL and Swift, long-term radio flux and structure monitoring, as well as optical observations by ground-based facilities.

The fast response ToO pointing by INTEGRAL provided $200 \mathrm{ks}$ of observations during the period 2008 August 9, 01:53 UT to August 11, 15:12 UT (revolution 711). However, PKS $1502+106$ was not detected (preliminarily) by the imager IBIS on board INTEGRAL. Extrapolating the X-ray flux observed by Swift, the hard-X-ray flux had likely already faded to slightly below the IBIS detection threshold in this epoch.

\subsection{Simultaneous X-ray and UV-Optical Observations and Results by Swift}

The Swift satellite (Gehrels et al. 2004) performed a ToO monitoring campaign of PKS $1502+106$ with daily snapshots from 2008 August 7 to 22. This quite long-term observing campaign by Swift allowed extended daily snapshots for about 16 days, using the three instruments onboard: the X-ray telescope (XRT) for the $0.2-10 \mathrm{keV}$ energy band, the Ultraviolet/Optical Telescope (UVOT) for multiband photometry, and the Burst Alert Telescope (BAT) for the $15-150 \mathrm{keV}$ hard X-ray band. BAT data were not used because of source confusion problems with Mkn 841 which is about a factor of 10 brighter than PKS $1502+106$ in the hard X-ray band. The 16 days of observations by Swift allow for cross-correlation studies between the $\gamma$-ray, $\mathrm{X}$-ray, and UV-optical bands during both the active flaring stage and the fading post-flare stage of PKS 1502+106.

The XRT was set in photon counting mode, and the data were processed by the xrtpipeline with the use of standard software (HEADAS software, v6.4) and standard filtering and screening criteria. The XRT events in the $0.3-10 \mathrm{keV}$ energy band were extracted using the XRTGRBLC FTOOL from circular regions centered on the source position with variable radii depending on the source intensity and applying correction for vignetting, PSF corrections and hot pixels and columns with the use of exposure maps. The XRT X-ray flux light curve is shown in the second panel of Figure 4. The $0.3-10 \mathrm{keV}$ count rate of PKS $1502+106$ measured by XRT was at a level 0.05 counts $\mathrm{s}^{-1}$ (from our data), up from a level of 0.02 counts s $^{-1}$ (archival past observations). The $0.3-10 \mathrm{keV}$ XRT 16-day long light curve obtained in 2008 August (Figure 4) shows an initial count rate of 0.05 counts $\mathrm{s}^{-1}$, and a gradual decay down to the level of about 0.02 counts $\mathrm{s}^{-1}$. The X-ray spectrum of each observation segment was fitted with an absorbed power law (Figure 5 and Table 2). Because of the low number of events from the source, events were not grouped and C-statistics was used, fixing the column density $N_{H}$ to the Galactic value $N_{H I}=2.19 \times 10^{20}$ $\mathrm{cm}^{-2}$ in that direction (in agreement with values used, e.g., in George et al. 1994; Akiyama et al. 2003), and using $z=1.839$. The error on the photon index and the flux $(0.3-10 \mathrm{keV})$ is large due to the low statistics. The background photons were selected

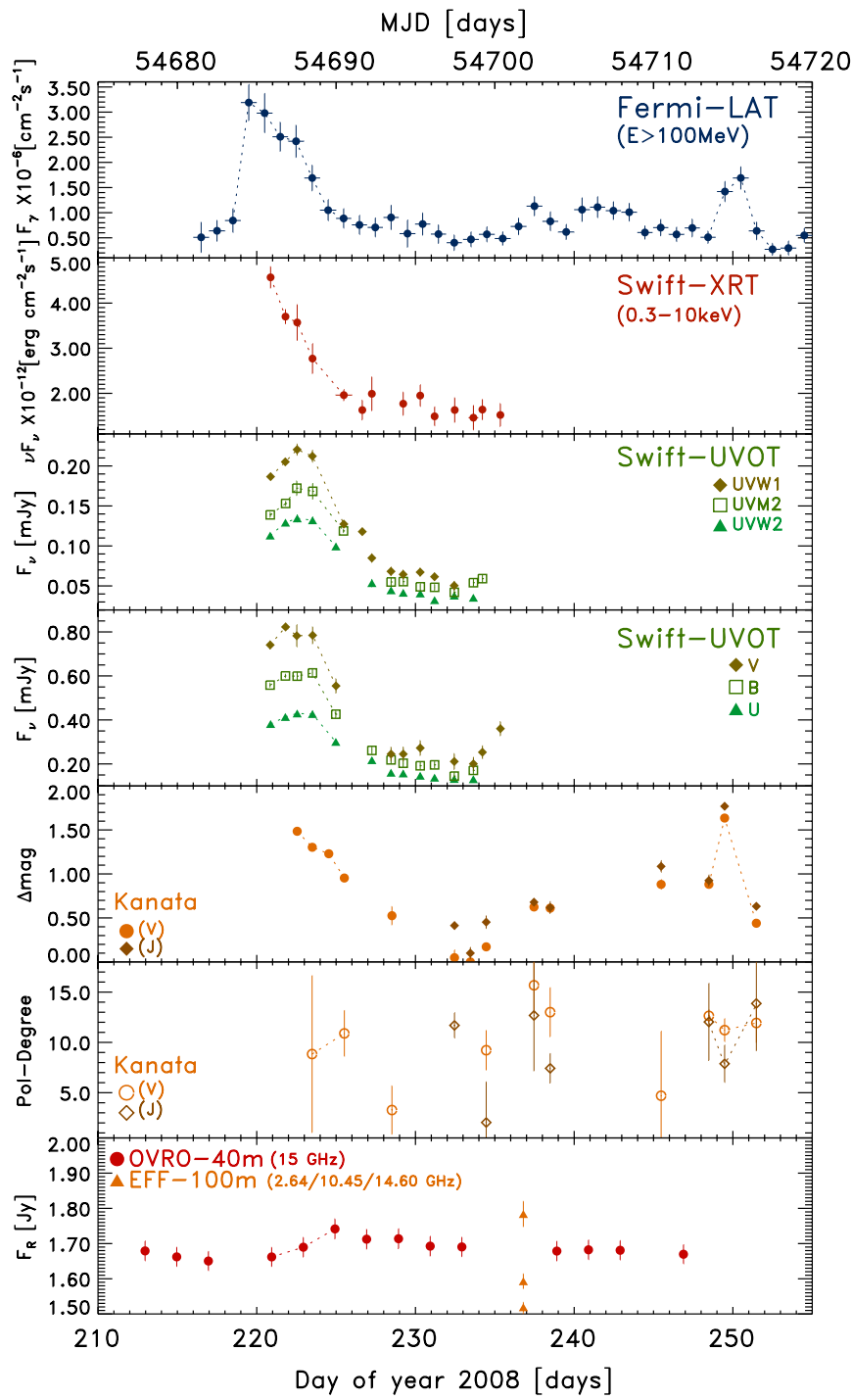

Figure 4. Simultaneous gamma-ray and multifrequency light curves obtained during the multiwavelength campaign of 2008 August triggered by the highenergy outburst discovered by Fermi LAT. The flux above $100 \mathrm{MeV}$, the X-ray flux (0.3-10 keV) by Swift-XRT, the six-band fluxes monitored by Swift-UVOT, the Kanata-TRISPEC differential relative magnitude light curves (optical $\Delta V$ and near-IR $\Delta J$ bands) and corresponding measures of the linear polarization degree, and the $15 \mathrm{GHz}$ radio light curve from OVRO $40 \mathrm{~m}$ are reported.

(A color version of this figure is available in the online journal.)

in a circular region close to the source. No significant photon index variation was observed between the high and the low state, while the count rate and flux did vary.

The Swift Ultraviolet/Optical Telescope (UVOT) photometry was done using the publicly available UVOT FTOOLS data reduction suite, and is in the UVOT photometric system described in Poole et al. (2008). The photometric data points were corrected for Galactic extinction using the dust maps of Schlegel et al. (1998) and the Milky Way extinction curve of Pei (1992). These simultaneous multiband optical and UV data show an increase of about 2 mag in all filters when compared with the past-years archival values (i.e., from about 19 to 17 in the $B$ band). The flux light curves in the six UVOT bands are shown in the third and fourth panel of Figure 4. These fluxes appear to be well correlated. A slight rise in flux of 3 days is observed in all the UVOT bands, followed by a fading similar to the flux decrease seen in $\gamma$-rays and $\mathrm{X}$-ray bands. If the time of the 


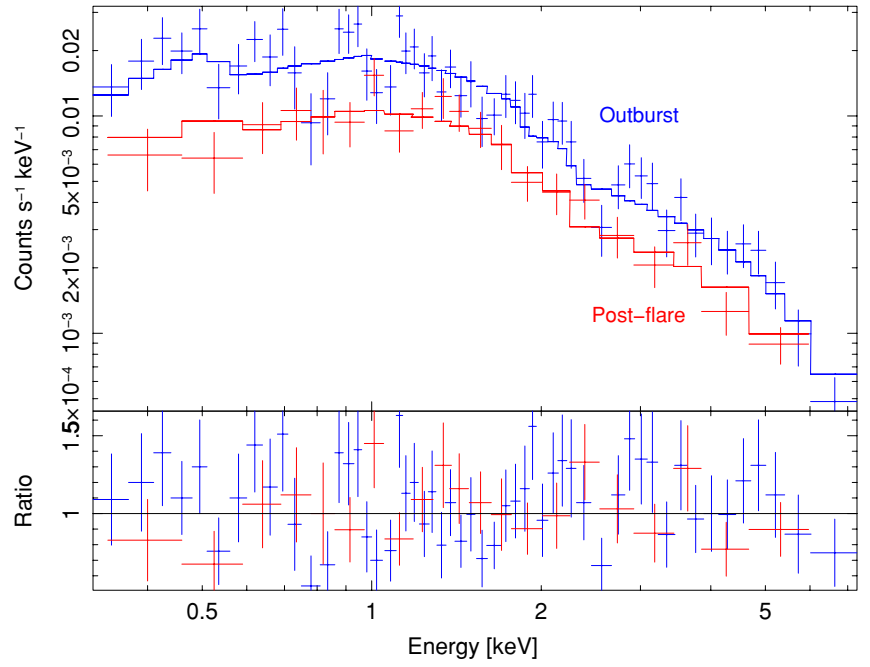

Figure 5. Swift-XRT combined $0.3-10 \mathrm{KeV}$ spectra of PKS $1502+106$ extracted for the high state (MJD: 54685-54689) and the subsequent low state (MJD 54690-54701), mapping the X-ray behavior simultaneous to the LAT flare and to the post-flare, relaxing activity and brightness.

(A color version of this figure is available in the online journal.)

observed UV and optical maximum is related to the flare activity at higher energies, this would imply an interesting time lag of about 4 days.

\subsection{Simultaneous Near-infrared and Optical Monitoring}

PKS $1502+106$ was also monitored in the optical $V$ and near-infrared $J$ bands with some photometric and polarimetric snapshots by the TRISPEC instrument attached to the $1.5 \mathrm{~m}$ "KANATA" telescope at the Higashi-Hiroshima Observatory, Japan (Watanabe et al. 2005; Uemura et al. 2008), within a twofold program of optical follow up for LAT flaring sources and regular monitoring of about 20 blazars. Imaging relative photometry was performed using some comparison stars in the same field, but due to the absence of an accurate calibration for this field we prefer to report only the relative magnitude difference $\Delta$ mag with respect to the minimum level (fifth panel of Figure 4). The optical and near-infrared band imaging photometry is performed simultaneously in TRISPEC with a unit of polarimetric sequence (consisting of successive exposures at four position angles of the half-wave plate, where a set of linear polarization parameters, $Q / I, U / I$, are derived).

These flux observations, performed for a longer interval with respect to the Fermi-Swift campaign (i.e., until 2008 September 22), show a high correlation between the $V$ - and $J$ band light curves and show an optical decay phase comparable to that observed in the UVOT photometric observations (fifth panel of Figure 4). Remarkably, a strong correlation with the LAT gamma-ray light curve is found, including the first (2008 September 4-7) of the possible minor flares occurring after the initial large outburst. The observation of a flare in the optical $(V)$ and near-IR $(J)$ bands, simultaneous with a second $\gamma$-ray flare have the twofold advantage of providing a validation of such a minor LAT flare as a real feature displayed by this blazar, and, even more crucially, in confirming the firm identification of the new gamma-ray source seen by Fermi with the blazar PKS 1502+106.

Comparing the Kanata-TRISPEC $V$-band and $J$-band colors, the $V-J$ color index varies between 2.05 and 1.69 (during the September 4-7 minor, rapid flare cited above). On the other
Table 2

Analysis Summary of the Simultaneous Data Obtained by the XRT Instrument on Board Swift

\begin{tabular}{lc}
\hline \hline \multicolumn{1}{c}{ Obs. ID (Date) } & Best-fit Model and Parameters \\
\hline All observations & Power law \\
(MJD 54685-54701) & $\Gamma_{X}=1.53_{-0.07}^{+0.06}$ \\
$t_{\text {exp }}: 52910 \mathrm{~s}$ & $\chi_{r}^{2}=1.05 / 80$ \\
& $F_{0.3-10 \mathrm{keV}}=1.9_{-0.11}^{+0.08} \times 10^{-12} \mathrm{erg} \mathrm{cm}^{-2} \mathrm{~s}^{-1}$ \\
\hline Outburst/high state & Power law \\
(MJD 54685-54689) & $\Gamma_{X}=1.54 \pm 0.08$ \\
$t_{\text {exp }}:$ 27680 s & $\chi_{r}^{2}=1.11 / 52$ \\
& $F_{0.3-10 \mathrm{keV}}=2.18_{-0.12}^{+0.11} \times 10^{-12} \mathrm{erg} \mathrm{cm}^{-2} \mathrm{~s}^{-1}$ \\
\hline Post-flare/lower state & Power law \\
(MJD 54690-54701) & $\Gamma_{X}=1.45_{-0.11}^{+0.12}$ \\
$t_{\text {exp }}:$ 25230 s & $\chi^{2}=0.76 / 32$ \\
& $F_{0.3-10 \mathrm{keV}}=1.39_{-0.12}^{+0.14} \times 10^{-12} \mathrm{erg} \mathrm{cm}^{-2} \mathrm{~s}^{-1}$ \\
\hline
\end{tabular}

hand, the degree $P$ of linear optical (in $V, J$ bands) polarization observed (sixth panel of Figure 4) remains rather scattered by error dispersion irrespective the flux level, even during the minor flare mentioned (the maximum degrees recorded during the monitoring were $P(V)_{\max }=15 \% \pm 3 \%$ and $P(J)_{\max }=$ $13 \% \pm 4 \%)$.

\subsection{Simultaneous Radio Flux-structure Data by Single-dish and VLBI Observations}

As part of an ongoing blazar monitoring program, the OVRO $40 \mathrm{~m}$ radio telescope has observed PKS $1502+106$ at $15 \mathrm{GHz}$ approximately every two days since mid-2007. Flux densities for the periods from 2008 July 26 to September 3 (MJD 5467354711) and 2008 October 23 to December 9 (MJD 5476254809) are shown in Figure 6. Flux densities were measured using azimuth double switching as described in Readhead et al. (1989) after peaking up on source. The relative flux density uncertainty for this source is dominated by a conservative $1.6 \%$ systematic error with a typical thermal error contribution of $5 \mathrm{mJy}$. Absolute flux density is calibrated to about $5 \%$ using the Baars al. (1977) model for 3C 286. This absolute uncertainty is not included in the plotted errors.

The measured flux densities in the MJD 54673-54711 (DOY 208-246) time period fit a $1.69 \mathrm{Jy}$ constant-flux model with $\chi^{2} /(N-1)=0.70(N=15$ data points $)$. This indicates no statistically significant variability in this time period. The beginning of a bright radio flare is apparent in the MJD 5476254809 (DOY 297-344) time period with an increase of at least $30 \%$ over the earlier mean flux density.

A less intensive monitoring at $37 \mathrm{GHz}$ was carried out with the $13.7 \mathrm{~m}$ radio telescope at Metsähovi Radio Observatory, Helsinki University of Technology, Finland. The flux density scale is set by observations of DR 21, and sources 3C 84 and 3C 274 are used as secondary calibrators. A detailed description on the data reduction and analysis can be found in Teräsranta et al. (1998).

The PKS $1502+106$ flare was also followed up by the Effelsberg $100 \mathrm{~m}$ radio telescope with four multifrequency radio spectra obtained on 2008 August 23, September 16, October 18, and December 6 (within the F-GAMMA project; see Fuhrmann et al. 2007). Each radio spectrum consists of simultaneous measurements at various frequencies between 2.6 and $42 \mathrm{GHz}$. The observations were performed using cross-scans in azimuth/ elevation with the number of sub-scans matching the source's brightness at the given frequencies. The data reduction was done 


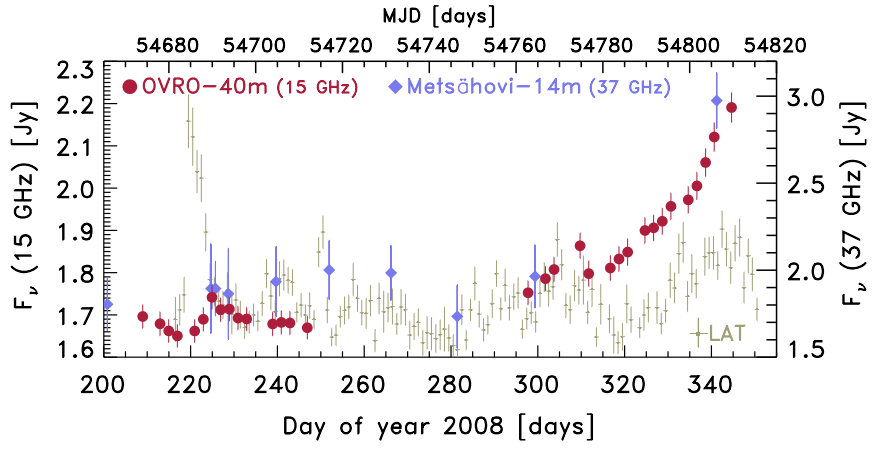

Figure 6. Long-term radio flux light curve at $15 \mathrm{GHz}$ obtained by the $40 \mathrm{~m}$ dish radio telescope of OVRO (filled circles), showing the rising part of a radio outburst started in late 2008 November, i.e., almost four months after the gamma-ray outburst detected by Fermi. The filled diamonds represent the flux measurements performed by the Metsähovi $14 \mathrm{~m}$ radio telescope at $37 \mathrm{GHz}$ (right $y$-axis scale), confirming the start of a radio outburst at a higher frequency. The scaled LAT daily light curve on the same period is reported for comparison (small light gray bars).

(A color version of this figure is available in the online journal.)

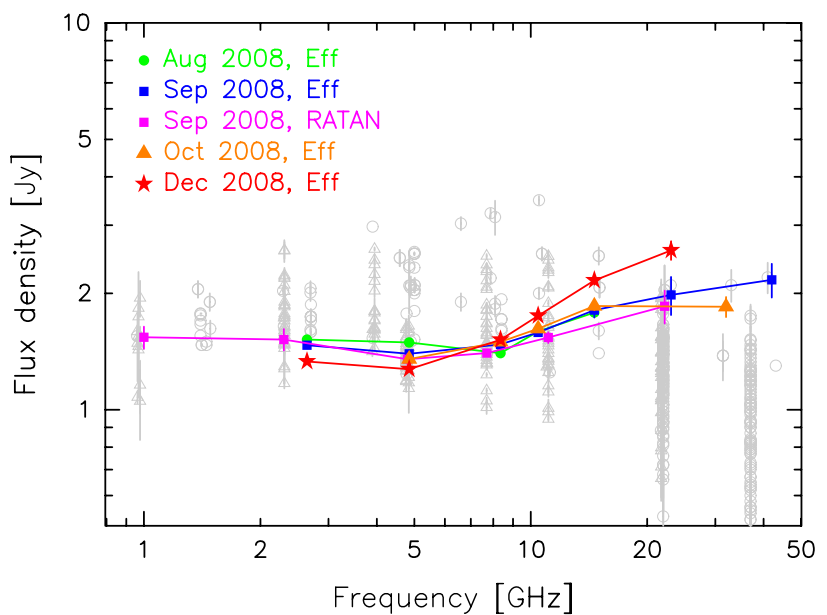

Figure 7. Variable broadband radio spectra observed with the Effelsberg $100 \mathrm{~m}$ and RATAN-600 radio telescopes simultaneous to the LAT data. Historical RATAN-600 data (gray open triangles) and archival data from the literature until 2008 March (gray open circles) are shown in the background for comparison.

(A color version of this figure is available in the online journal.)

using standard procedures described in Fuhrmann et al. (2008); Angelakis et al. (2008).

Other radio observations are available at six frequencies between 1 and $21.7 \mathrm{GHz}$, thanks to the $600 \mathrm{~m}$ ring transit radio telescope of the Russian Academy of Sciences RATAN-600 (Korolkov \& Parijskij 1979), which observed the source on 2008 September 10, 26, and October 2. A weighted average of these three observations, is presented in Figure 7. Previous RATAN-600 data which cover the period between 1997 and 2008 March are also shown for comparison. The observing methods, the data processing, and the amplitude calibration is described in Kovalev et al. (1999).

All single-dish spectra obtained with the Effelsberg $100 \mathrm{~m}$ and RATAN-600 radio telescopes are presented in Figure 7. Here, no indication of a flare or strong difference/variability between August and September can be noted. However, the 2008 December spectrum shows the beginning of a bright radio flare with a clear spectral steepening toward higher frequencies $(v>10 \mathrm{GHz})$. This is in good agreement with the strong flux density increase seen in the $15 \mathrm{GHz}$ light curve during November/December (Figure 7).

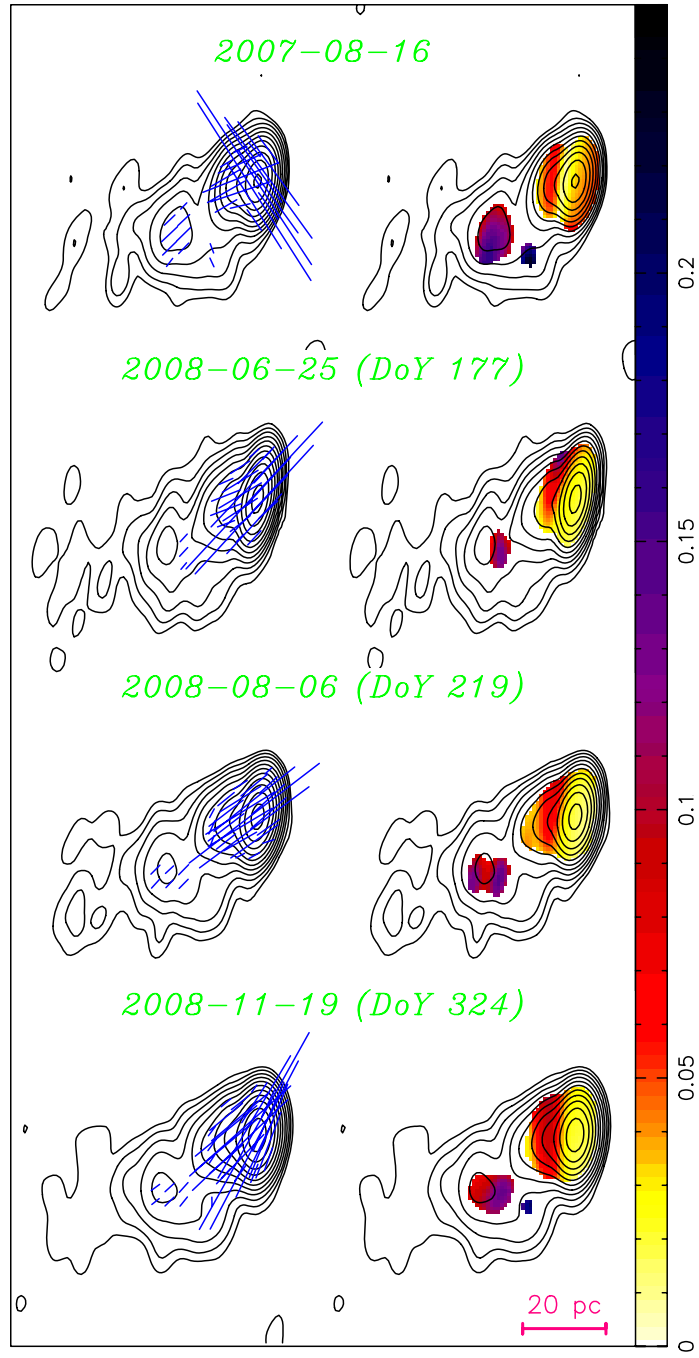

Figure 8. Total intensity and linear polarization images observed by the VLBA at $15 \mathrm{GHz}$ as part of the large Fermi-supporting MOJAVE program. Naturally weighted total intensity images are shown by black contours. The contours are in successive powers of two times the base contour level of $1.0 \mathrm{mJy}^{\mathrm{beam}}{ }^{-1}$. Electric polarization vector directions are indicated on the left-hand side by blue sticks, with their length being proportional to the polarized intensity. Linear fractional polarization is shown on the right-hand side overlaid according to the color wedge.

(A color version of this figure is available in the online journal.)

Detailed radio images at sub-milliarcsecond scale of the PKS 1502+106 superluminal jet were obtained during three epochs in 2008 with Fermi already in orbit: on June 25, August 6 (during the maximum peak of the $\gamma$-ray outburst), and November 19. These observations were performed as part of the MOJAVE monitoring program conducted with the VLBA at $\lambda=2 \mathrm{~cm}$ (Lister \& Homan 2005; Lister et al. 2009a) and provided useful high-resolution total intensity and linear polarization images. These VLBA images close to the $\gamma$-ray flare are reported and compared to the map obtained one year earlier in Figure 8 . The highest integrated flux density value since the beginning of the $2 \mathrm{~cm}$ VLBA monitoring in 1997 (Kellermann et al. 2004) was measured on 2008 November 19 (DOY 324) as $F_{15 \mathrm{GHz}}=2.0 \mathrm{Jy}$, with a peak intensity of $1.6 \mathrm{Jy}$ beam $^{-1}$. These values are significantly higher than the typical level of $1.3 \mathrm{Jy}$ reported in the program (Kovalev et al. 2005; Lister et al. 2009a, 2009b) and indicate a radio flare happening in the source VLBI core. The core flux density and 
brightness temperature raised to higher values as well which means that the flare happens in the VLBI core, as expected. These findings are in good agreement with the single-dish results presented above (Figures 6 and 7). The second relevant feature is the direction of the electric vector position angle (EVPA) in the core region, which rotated between the 2007 and 2008 epochs by $90^{\circ}$ most probably indicating an opacity change-a precursor of an outburst in the VLBI core.

In summary, our single-dish and VLBI radio monitoring of PKS 1502+106 simultaneous to the Fermi LAT observations has revealed (1) no significant radio $15 \mathrm{GHz}$ variability during the strong LAT $\gamma$-ray flare seen in 2008 August and (2) a strong radio flare which becomes clearly visible at $v>10 \mathrm{GHz}$ during 2008 October/November (Figure 6) with a rise phase lasting for at least 20 days. If this flaring behavior is associated with the bright $\gamma$-ray flare of 2008 August, a delay of more than three months $(\sim 98$ days if the lag between the starting days of the $\gamma$-ray outburst, DOY 218.2, and the $15 \mathrm{GHz}$ outburst, DOY 316.7 , is considered) could be explained by opacity effects in the core region of the source (e.g., Aller et al. 1999). Although we cannot exclude the possibility of radio activity at $15 \mathrm{GHz}$ during the OVRO $40 \mathrm{~m}$ observations outage (2008 September 3-October 23) that might also be associated with the gamma-ray flare of 2008 August, OVRO data before and after the outage as well as September and October Effelsberg $100 \mathrm{~m}$ data are consistent with very little change in the $15 \mathrm{GHz}$ flux over this time period. The three flux measures at $37 \mathrm{GHz}$ obtained at the Metsähovi radio observatory during the OVRO outage, confirms this trend with very little variability during this period. However, the observed radio flare could also be associated with, e.g., the more recent, prominent variability seen in the LAT $\gamma$ ray data during November/December (Figure 2; DOY 320333). A more detailed analysis of such possible correlations and the source's overall radio $/ \gamma$-ray behavior seen with LAT and simultaneous radio observations over a longer period of time will be the subject of a subsequent work.

\section{ARCHIVAL MULTIWAVEBAND DATA}

A full multiwavelength analysis dedicated on PKS 1502+106 is available only from George et al. (1994), where old archival and broadband radio to X-ray data obtained in 1993-1994 were presented. Data and analysis on PKS $1502+106$ reported in other papers are mostly limited to the radio regime. In order to compare our multifrequency findings with the past, and to form a more complete characterization of this blazar, we briefly present here, for the first time, results from unpublished past observations by the INTEGRAL, XMM-Newton, Swift, and Spitzer space telescopes performed in 2001, 2005, and 2006.

\subsection{INTEGRAL Observations in 2006}

The sky region containing PKS $1502+106$ was observed in 2006 by IBIS (83 ks, MJDs 53760.4 to 53762.4, 2006 January 25-27), and a new soft $\gamma$-ray source (IGR J15039+1022) was detected with a flux density of $1.6 \mathrm{mCrab}$ in the $18-60 \mathrm{keV}$ energy range (corresponding to $1.2 \times 10^{-11} \mathrm{erg} \mathrm{cm}^{-2} \mathrm{~s}^{-1}$, see ATel \#1652). This IBIS source was identified with Mkn 841, a Seyfert galaxy known to display a well-detected high-energy cutoff around $100 \mathrm{keV}$ (Petrucci et al. 2002), making it unlikely to emit in the $\gamma$-ray domain. The angular distance of PKS 1502+106 from IGR J15039+1022, 11', points to a clear non-detection during this 2006 January INTEGRAL observation, while a $2 \sigma$ upper limit for PKS $1502+106$ of
Table 3

Analysis Summary of the EPIC-MOS Instrument Observations (2001 January and 2005 July) on Board of XMM-Newton

\begin{tabular}{lc}
\hline \hline \multicolumn{1}{c}{ Obs. ID (Date) } & Best-fit Model and Parameters \\
\hline ObsID 0070740101 & Power law \\
(2001 Jan 13, 09:20 UTC) & $\Gamma_{X}=1.6 \pm 0.2$ \\
& $\chi_{r}^{2}=1.69 / 11$ \\
& $F_{0.2-10 \mathrm{keV}}=3.5 \times 10^{-13} \mathrm{erg} \mathrm{cm}^{-2} \mathrm{~s}^{-1}$ \\
\hline ObsID 0070740301 & Power law \\
(2001 Jan 14, 00:30 UTC) & $\Gamma_{X}=1.7 \pm 0.2$ \\
& $\chi_{r}^{2}=1.27 / 17$ \\
& $F_{0.2-10 \mathrm{keV}}=6.8 \times 10^{-13} \mathrm{erg} \mathrm{cm}^{-2} \mathrm{~s}^{-1}$ \\
\hline ObsID 0112910201 & Power law \\
(2001 Jan 13, 04:58 UTC) & $\Gamma_{X}=1.6 \pm 0.2$ \\
& $\chi_{r}^{2}=1.05 / 8$ \\
& $F_{0.2-10 \mathrm{keV}}=3.6 \times 10^{-13} \mathrm{erg} \mathrm{cm}^{-2} \mathrm{~s}^{-1}$ \\
\hline ObsID 0205340401 & Power law \\
(2005 Jul 17, 06:32 UTC) & $\Gamma_{X}=1.69 \pm 0.08$ \\
& $\chi_{r}^{2}=0.99 / 58$ \\
& $F_{0.2-10 \mathrm{keV}}=11.0 \times 10^{-13} \mathrm{erg} \mathrm{cm}^{-2} \mathrm{~s}^{-1}$ \\
\hline
\end{tabular}

$0.7 \mathrm{mCrab}$ in the range $18-60 \mathrm{keV}\left(0.52 \times 10^{-11} \mathrm{erg} \mathrm{cm}^{-2} \mathrm{~s}^{-1}\right)$ is inferred.

\subsection{XMM-Newton and Swift}

Four serendipitous, unpublished XMM-Newton X-ray observations of PKS $1502+106$ by the EPIC (MOS detector only; Strüder et al. 2001) camera are available as the source was in the frame of the target Seyfert galaxy Mkn 841. PKS 1502+106 was always on the border of the MOS chips and out of the PN frame, and therefore subject to low X-ray statistics, regardless of its intrinsic brightness. The four X-ray EPIC-MOS observations (three in 2001 and one performed in 2005 July 17; see Table 3 for an analysis summary) do not show variations in the $0.2-10$ $\mathrm{keV}$ photon index, while the $0.2-10 \mathrm{keV}$ flux intensity varied by a factor of a few (in the range (3.5-6.8) $\times 10^{-13} \mathrm{erg} \mathrm{cm}^{-2}$ $\mathrm{s}^{-1}$ in 2001, comparable to the lower states observed by $A S C A$, and a mildly active state with $1.1 \times 10^{-12} \mathrm{erg} \mathrm{cm}^{-2} \mathrm{~s}^{-1}$ in 2005 July, Table 3).

PKS 1502+106 was also observed twice in the past with the Swift-XRT as a fill-in target (Target ID: 36388), showing a fainter X-ray flux $\left(0.02\right.$ counts $\left.\mathrm{s}^{-1}\right)$ than the flux recorded in the 2008 August campaign observations ( 0.05 counts $\left.\mathrm{s}^{-1}\right)$.

\subsection{Spitzer Observations and the Multifrequency Behavior on 2005 July-August}

In the past, only upper limits in the far-/near-infrared bands by IRAS were available for PKS 1502+106 (Neugebauer et al. 1986; Figures 9 and 11). PKS 1502+106 was observed serendipitously in the mid-infrared band for the first and only time by Spitzer on 2005 August 13, 09:10-09:18 UT (PID 117, AOR Request Key 5011456). The Infrared Spectrograph (IRS; Houck et al. 2004), low-resolution ( $R=60-130)$ module, recorded the mid-IR spectrum from 5-14 $\mu \mathrm{m}$ (shown in Figure 9 with the optical SDSS spectrum and near-IR photometric data point). High-resolution IRS module spectra were also taken, but could not be used since there were no accompanying background observations. The Short-Low (SL) co-added two-dimensional spectra were reduced using the standard Spitzer IRS pipeline (version S17.2). Background was subtracted using the two nod positions along the slit. The spectra were extracted and fluxcalibrated with SPICE version 2.1.2, in a standard, expanding 


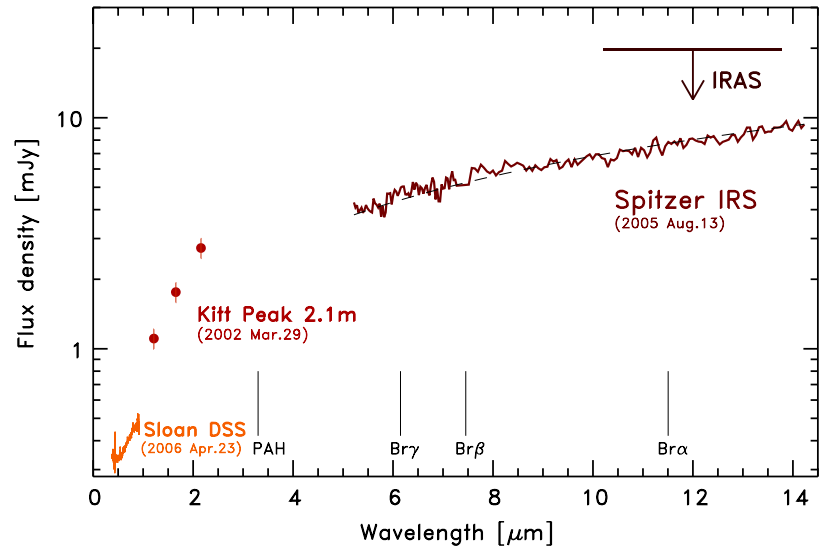

Figure 9. Unique observed mid-IR spectrum in the range 5-14 $\mu \mathrm{m}$ obtained by the Spitzer Infrared Spectrograph (IRS) low-resolution $(R=60-130)$ module, on 2005 August 13. The position of redshifted Brackett emission lines and PAH line are indicated, even if they are not detected in the IRS spectrum that is consistent with a simple power-law model. In addition, the optical spectrum by the SDSS on 2006 April 23, and high precision $\mathrm{JHK}_{\mathrm{S}}$ photometric flux measurements of the Kitt Peak National Observatory (KPNO) $2.1 \mathrm{~m}$ telescope of 2002 March 29 are also reported.

(A color version of this figure is available in the online journal.)

point-source aperture. The two spectral orders match well at $7 \mu \mathrm{m}$, indicating a well-pointed observation. The mid-IR continuum of PKS $1502+106$ rises to the near infrared and appears to be rather featureless, consistent with pure synchrotron emission (a power-law $F_{v} \propto v^{-0.9}$ in the 5-14 $\mu \mathrm{m}$ range; Figure 9). The wave-like deviation of the data can be simply explained as wavelength-dependent spectrograph slit losses, while the redshifted Brackett emission line series (like the $3.3 \mu \mathrm{m}$ PAH feature) falling in this wavelength range are indicated, but they are not well detected in the spectrum. This IRS spectrum is similar to other blazars which have been observed by Spitzer, including BL Lac and 3C 454.3 (Leipski et al. 2009; P. Ogle et al. 2010, in preparation), while the near-IR $(J, H, K)$ flux data from the Kitt Peak National Observatory (KPNO) $2.1 \mathrm{~m}$ telescope (Watanabe et al. 2004) reported in the same figure indicate a lower flux state and steeper $H-K_{\mathrm{S}}(1.65-2.15 \mu \mathrm{m})$, spectral index $\left(\alpha_{\left(H-K_{\mathrm{S}}\right)}=1.66\right)$.

The 2005 July-August spectral energy distribution (SED) assembled with these XMM-Newton (2005 July 17) and Spitzer (2005 August 13) observations (joined with a couple of radiooptical observations in these two months by the Metsähovi, RATAN, and Catalina observatories) is consistent with a low or mildly active stage that can be explained by a simple SSC model (inset plot in Figure 11). Based on these data there are therefore no hints for a deviation from an SSC scenario in this flat spectrum radio quasar. In any case, this SED cannot provide significant constraints, because it contains observations obtained over about two months and does not include any $\gamma$-ray data, aside from the older (1992) EGRET upper limit (calculated with the prescriptions in Thompson et al. 1996).

XMM-Newton observations pointed out a $(0.2-10 \mathrm{keV})$ photon index that shown almost no variations, with values $\left(\Gamma_{X} \sim\right.$ $1.7)$ in agreement with the previous $X$-ray observations performed by ROSAT and ASCA (George et al. 1994; Watanabe et al. 2004). The integrated X-ray flux $F_{0.2-10 \mathrm{keV}}=1.0 \times 10^{-12}$ suggests a mildly active state during this observation, comparable to the Swift XRT spectrum observed in the days soon after the LAT outburst (red filled symbols, data points in Figure 11). More complicated and possibly more accurate emission models, beyond the SSC, can be therefore investigated for the first time only thanks to the Fermi multifrequency campaign of 2008 August, whose SED is described in the following section. The Swift XRT simultaneous spectrum of 2008 August has a slightly harder spectrum with respect to these archival XMM-Newton observations, while the XRT flux was about one order of magnitude greater than the flux observed in the past by XMM-Newton, $A S C A$, and ROSAT.

\section{DISCUSSION}

\subsection{Gamma-ray Outburst and Longer Term Variability}

During the first several months of the LAT survey, PKS 1502+106 was one of the brightest, as well as the most luminous, blazar in the MeV-GeV band. The threefold flux increase in $\lesssim 12$ hr between 2008 August 5 and 6 (DOY 218219; Figure 2) constrains the rest-frame size $\left(R^{\prime}\right)$ of the flaring region: $R^{\prime} \leqslant c \Delta t \mathcal{D} /(1+z) \simeq 6.8 \times 10^{15} \mathrm{~cm}$ (where $\mathcal{D}=1 /(\Gamma(1-\beta \cos \theta))$ is the macroscopic Doppler factor, $\Gamma$ is the bulk Lorentz factor, and $\theta \simeq 1 / \Gamma$ is the angle of sight). The value $\mathcal{D} \simeq \Gamma \simeq \beta_{\text {app }}=14.8$ is assumed from MOJAVE VLBA measurements (Lister et al. 2009b), where $\beta_{\text {app }}$ is the kinematic apparent jet speed in units of $c$. In this high-speed regime an upper limit on the viewing angle can also be estimated: $\theta<2 \arctan \left(1 / \beta_{\mathrm{app}}\right)=7$.7. This is consistent with an independent estimation of the Doppler factor based on the $37 \mathrm{GHz}$ flux variability, made in Hovatta et al. (2009): a factor $\mathcal{D}_{\text {var }}=12$ and $\beta_{\text {app_var }}=14.6$, with brightness temperature $T_{\mathrm{br}}=8.7 \times 10^{13} \mathrm{~K}$, and angle of sight $\theta=4.7$.

Assuming the "concordance" cosmology (Section 1 and $z=1.839$ ), the luminosity distance of PKS $1502+106$ is $d_{L}=$ $14.2 \mathrm{Gpc}$, and the inferred, apparent and isotropic, monochromatic luminosity at $E_{0}=100 \mathrm{MeV}$ of PKS $1502+106$ during the outburst phase is $L_{E>100} \simeq 4 \pi d_{L}^{2} \cdot(\Gamma-1) E_{0} F_{E>E_{0}} \simeq 1.1 \times$ $10^{49} \mathrm{erg} \mathrm{s}^{-1}$, where the average flux $F_{E>E_{0}}=2.91 \times 10^{-6} \mathrm{ph}$ $\mathrm{cm}^{-2} \mathrm{~s}^{-1}$ in the outburst interval (DOY 2008: 218.95-224.0) is used. The bolometric luminosity is expected to be even higher than this value, since the measured LAT spectrum appears to be beyond the peak of the high-energy component, and therefore this LAT blazar has probably one of the highest $L_{\gamma} / \Delta t$ ratios $\left(2.5 \times 10^{43} \mathrm{erg} \mathrm{s}^{-2}\right)$ known in the $\mathrm{MeV}-\mathrm{GeV}$ regime.

Relativistic motion provides a solution for the problem of intrinsic excess absorption by pair-production in powerful $\gamma$-ray sources like PKS 1502+106 (see, e.g., Mattox et al. 1993; Madejski et al. 1996) which have a significant $L_{\mathrm{BLR}}$. Adopting the flux tripling timescale of the outburst rise (i.e., $\Delta t=12 \mathrm{hr}$ ), and the outburst state averaged X-ray flux $\left(F_{0.3-10 \mathrm{keV}}=2.18_{-0.12}^{+0.15} \times 10^{-12}\right.$; Table 2$)$ at the observed photon frequency $v_{X}=10^{18} \mathrm{~Hz}$, the minimum Doppler factor $\mathcal{D}$ required for the photon-photon annihilation optical depth to be $\tau_{\gamma \gamma} \leqslant 1$ can be estimated. Using the derived relation $1=\tau_{\gamma \gamma} \simeq \sigma_{T} d_{L}^{2} F_{X} /\left(3 \Delta t c^{2} E_{X} \mathcal{D}^{4}\right)$ and taking the region size $R=c \Delta t \mathcal{D} /(1+z)$, the source-frame photon energy $E_{X}^{\prime}=(1+z) h v_{X} / \mathcal{D}$ and the intrinsic X-ray luminosity $L_{X}^{\prime}=$ $4 \pi d_{L}^{2} \mathcal{D}^{-4} F_{X}$ we obtain $\mathcal{D} \gtrsim 7.7$ (omitting the requirement of co-spatiality of the $\mathrm{X}$-ray and $\gamma$-ray emission regions relaxes this limit). This is in agreement with the values found from radio flux structure observations and with the SED modeling parameters found.

The asymmetry of the August 5-6 $\gamma$-ray outburst can suggest a more complex emission geometry than a simple one-zone model. The temporal structure- $\sim 0.5$ day rise, followed by a $\sim 4.5$ day decay where a $\sim 2.5$ day intermediate-level plateau is 
likely observed-implies particle acceleration and cooling times that are greater than the light crossing time, i.e., $t_{\text {inj }}, t_{\text {cool }}>R / c$, where these quantities are evaluated in the jet comoving frame. A synchrotron self-Compton (SSC) emitting blob in the jet should be relatively confined $(<0.01 \mathrm{pc})$, although relativistic beaming would permit the region to be as much as an order of magnitude larger. The hinted intermediate plateau could mark a twofold active region, and two SSC emitting components. Descriptions making use of a multi-zone SSC or multi-emission component SSC (second-order, superquadratic components) are reported, e.g., in Sokolov \& Marscher (2005); Georganopoulos et al. (2006); Graff et al. (2008). On the other hand, if the injection of relativistic electrons is impulsive and repeated, some single-zone SSC models already predict plateaus during an outburst (see, e.g., Chiaberge \& Ghisellini 1999; Böttcher \& Chiang 2002; Sokolov et al. 2004). At lower frequencies (IR-optical-UV), where cooling times are longer, the electron distributions corresponding to different injections can build up, and the memory of the individual injection phases can be lost, providing a smoother decay without intermediate plateaus (as shown in X-ray, optical-UV light curves by Swift; Figure 4). Apparent delays (like the 4 day lag hinted by UVOT data) can also be explained within this scenario.

On the other hand, this asymmetric (fast rise, slower decay) shape of the $\gamma$-ray outburst can also be an evidence for a dominant contribution by Comptonization of photons produced outside the jet (Sections 5.3 and 5.4) during this event, as predicted, e.g., in Sikora et al. (2001). Gamma-ray flares produced by short-lasting energetic electron injections and at larger jet opening angles are predicted to be more asymmetric showing much faster increase than decay, the latter determined by the light travel time effects.

The "harder when brighter" trend of the gamma-ray photon index during the outburst (right inset panel of Figure 2), hints a narrow hysteresis evolution of the spectral index against the flux, a signature produced by non-thermal cooling and high- to low-energy propagation of the electron injection rate (Kirk et al. 1998; Georganopoulos \& Marscher 1998; Böttcher \& Chiang 2002). The photon index extracted with a power-law model over the daily bins was found quite scattered irrespective of the flux level in the remaining part of the light curve following the outburst.

The outburst of 2008 August appeared to have ignited an enduring relaxing state of $\gamma$-ray brightness and activity, during the following four months (Sections 2.3 and 5.1). The $1 / f^{a}$ (with $a \sim 1.3$ ) PSD points out a general fluctuation mode placed between a pink-noise (flickering) and a randomwalk (Brownian motion or brown noise), staggered by some rapid flares, similar to the long-term variability of blazars observed in radio and optical wavebands (e.g., Hughes et al. 1992; Ciaramella et al. 2004; Teräsranta et al. 2005; Ciprini et al. 2007b; Hovatta et al. 2007). In contrast, this variability mode is rather different than the full Brownian regime shown by the short-term (intra-hour resolution) light curve of the large TeV outburst of PKS 2155-304 (Aharonian et al. 2007). This PSD indicates the occurrence frequency of a specific variation is inversely proportional to its strength, as found in processes driven by stochastic relaxation, and rapid flares/ outbursts (phenomenologically called intermittence), are not occasional events produced by physical processes of different nature with respect to the mechanism responsible for the long-term flickering, but can be considered as statistical tails of the same dynamic process, possibly connected to disk or jet instabilities, to viscosity and magnetic turbulence, or to inhomogeneities and shocks (e.g., Begelman et al. 1984). Even in the case of quite nonhomogeneous structures, the jet is seen under a small viewing angle $(\theta<7.7$ here), therefore flaring events and variability trends are the result of emission components originating from different regions, excluding, in most cases, causality.

\subsection{Gamma-ray and Radio Connection}

The absence of significant radio $15 \mathrm{GHz}$ and $37 \mathrm{GHz}$ variability before, during and immediately after the LAT outburst (Figure 6), and strictly correlated to the near-IR to $\gamma$-ray outburst, has consequences for opacity at lower radio frequencies. However, the beginning of the strong radio flare seen in the 2008 October-December data of Figures 6 and 7 could be associated with the period of increased $\gamma$-ray activity seen after $\sim$ DOY 330 (2008 November 25, Figure 2). If the beginning of the radio outburst in Figure 6 is associated with the LAT outburst, this would imply a delay of more than about 98 days (Section 3.3), meaning that the radio emission region becomes transparent at the $\mathrm{cm}$ and longer wavelengths significantly after the outburst seen at wavelengths where the source is truly optically thin $(\mathrm{mm}$, IR, optical bands).

The rotation direction of the EVPA in the milliarcsecondscale core of PKS $1502+106$ between 2007 and 2008 was a possible precursor signature of an outburst that occurred in the core (Figure 8). The electric polarization vectors appear well aligned to the jet axis in the 2008 June 25 map and even more in the 2008 August 6 map. This latter map represents an unprecedented example of radio-structure snapshot truly simultaneous to the peak of a MeV-GeV outburst (Figure 8). About three months after the outburst, on 2008 November 11, the alignment is again decreasing. These interesting findings are in agreement with the scenario that assumes very bright $\gamma$-ray flares occurring after the ejections of superluminal radio knots, with accompanying increases in polarized radio flux, and a field ordering and alignment with respect to the jet axis. A correspondence between variations in polarization direction and intensity in different bands at parsec scales can help localize the primary site of the high-energy emission. We found that the prominent $\gamma$-ray outburst and the possible three-month delayed radio-outburst are likely produced in the core of the parsec-scale jet of PKS 1502+106. More details on connections between VLBI radio structures and high-energy emission, and their interpretation are reported recently in Jorstad et al. (2007), D'Arcangelo et al. (2007), Marscher et al. (2008), and Kovalev et al. (2009). To test if this is indeed the case for the discussed gamma-ray flare, VLBA monitoring of $1502+106$ is continued at 15 and $43 \mathrm{GHz}$.

\subsection{Gamma-ray, X-ray, and UV-optical Cross Correlations}

During the EGRET era, a similar degree of simultaneous $\mathrm{X}$-ray and $\gamma$-ray monitoring was achieved, for instance, for the blazar 3C 279 (Wehrle et al. 1998; Hartman et al. 2001), a typical $\gamma$-ray powerful FSRQ resembling PKS $1502+106$. The correlation found in PKS $1502+106$ between the $\gamma$-ray flux and the X-ray and UV-optical emission during the 16 days of Swift follow up, is evidenced by the multipanel and multifrequency light curves in Figure 4. The apparent linear correlation between the emission in the LAT band and the X-rays (Figure 10, left panel) suggests that the observed X-ray and $\mathrm{MeV}-\mathrm{GeV}$ photons may be part of a single SSC component (continuous line, labeled 

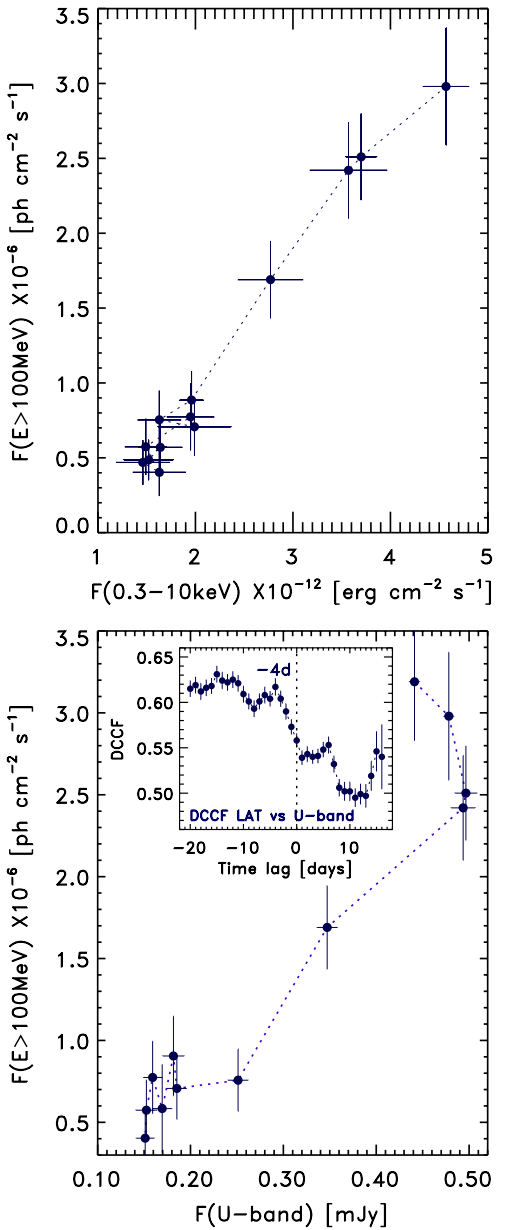

Figure 10. Top panel: the gamma-ray flux measured by Fermi LAT vs. the X-ray flux measured by Swift-XRT. The cross-correlation without lags is well displayed. Bottom panel: the LAT gamma-ray flux vs. the UV flux measured by $S$ wift-UVOT in the representative $U$ band, and the discrete cross-correlation function diagram (DCCF, inset plot) between the gamma-ray flux and the $U$ band flux. Here a clear correlation with a 4 day delay of the UV emission flare with respect to the gamma-ray flare is suggested.

(A color version of this figure is available in the online journal.)

"SSC2," on Figure 11). This could also explain the nearly constant X-ray $(0.3-10 \mathrm{keV})$ spectral index observed during the outburst with respect to the post-flare phase.

The $\gamma$-ray peak power observed during the outburst was $v F_{v} \sim 10^{49} \mathrm{erg} \mathrm{s}^{-1}$, and decreased to less than about $2 \times$ $10^{48} \mathrm{erg} \mathrm{s}^{-1}$ in the post-flare period, meaning a difference of at least a factor of $\sim 6$ (Figure 11). The difference between these two emission states in X-ray output was instead a factor of $\sim 2.5$, and a factor of $\sim 3.5$ in the optical-UV band (frequencies above the synchrotron peak). These sub-quadratic differences between the outburst and the subsequent state could be still described by SSC descriptions. The strict correlation between the X-ray flare and the $\gamma$-ray outburst supports, at least, the dominance in the SED (Figure 11) of the SSC, in-jet, emission from radio to X-ray bands in agreement with results detailed in Sikora et al. (2001). A simple single-zone SSC model has nevertheless problems explaining and reproducing the large $\gamma$ ray dominance observed during the outburst, and could require very sub-equipartition magnetic fields.

Figure 4 and the bottom panel of Figure 10 show the flux monitored by UVOT ( $U$-band reported there, but similar results are found in the other five filters), indicating the same strong correlation with respect to $\gamma$-rays, but with a possible time lag of about 4 days (also hinted at by the peak on the discrete cross-correlation function, DCCF, inset plot). This possible time lag can be reasonable only by assuming that the optical-UV brightness during the start and rise of the gamma-ray outburst (i.e., during DOY 216-219) was comparable or lower than the flux observed during the first UVOT observation (performed on DOY 220.8). Relative delays between the synchrotron (our Swift UVOT data) and the inverse Compton (IC; our Swift XRT and Fermi LAT data) counterparts, are dominated by energy stratification and geometry of the emitting region. In SSCdominated zones the synchrotron emission is co-located with the IC production site, and such 4 day optical-UV to $\gamma$-ray lag would depend on light-travel time effects in the emitting region (characterized by size $R$ and viewing angle $\theta$ ), and by the particle cooling time and decay time of the synchrotron and IC emissions. When the region is not so compact, both decay times can be comparable to the apparent light crossing time $R / c$, and significant shifts between light curves at these different energy bands are expected. Synchrotron flares (our opticalUV data) can be delayed with respect to the IC $\gamma$-ray flares (e.g., Chiaberge \& Ghisellini 1999 and Sokolov et al. 2004). Rather long time lags such as this could also be explained by a prolonged disturbance traveling down the jet. The disturbance, being radially inhomogeneous in both density and velocity, could induce shocks and collisions leading to the formation of two adjacent emission zones with similar properties (multizone SSC), thus explaining the flare shape asymmetry and the intermediate-level plateau during the decay (Section 5.1).

Unlike SSC emission, outbursts dominated by IC external radiation involve a constant field of seed photons, and are not delayed by light-travel time of photons. The resulting frequency stratification behind the shock front could extinguish an outburst first at the highest energies, then progressing to lower frequencies as time advances (Sokolov \& Marscher 2005), and energy-dependent radiative losses induce delays in the declining part of the emission produced by the lower energy particles. The variability patterns shown by our data could rule out radiative cooling as the only mechanism for causing the delay, since the rising part of the optical emission follows the rising part of the $\gamma$ rays. In addition, the decay timescale in the UVOT data seems comparable to that seen in the LAT, and not longer as required by energy-dependent dominated cooling. A reasonable mixture of SSC and an extra contribution by Comptonization of the BLR photon field (external jet origin) by the same population of energetic electrons (ERC; e.g., Sikora et al. 1994, 2001; Dermer \& Schlickeiser 2002; Ghisellini \& Tavecchio 2009) could be more adequate to explain also the SED, and such $\mathrm{SSC}+\mathrm{ERC}$ scenario is still compatible with the X-ray to $\gamma$-ray correlation found in PKS 1502+106.

As seen in Section 3.2, the first of the fast, minor $\gamma$-ray flares occurring in 2008 September (DOY 248-251) after the big outburst, was well observed by the Kanata-TRISPEC telescope in both $J$ and $V$ bands, with no significant time lag. The lower intensity and duration of the flare, the limited temporal resolution of the data, and a possible dominance of the SSC process during this episode can explain this difference with respect to August's big outburst. The match between the MeV$\mathrm{GeV}$ and optical-near-IR flare, was also crucial for the firm identification with PKS 1502+106 (Section 2.2), and was a reasonable confirmation of the source-intrinsic nature of this variation seen in the LAT light curve (Figures 2 and 4). These $V$ and $J$ flux measurements, obtained after the conclusion of the outburst phase but on a period longer than the Swift monitoring 


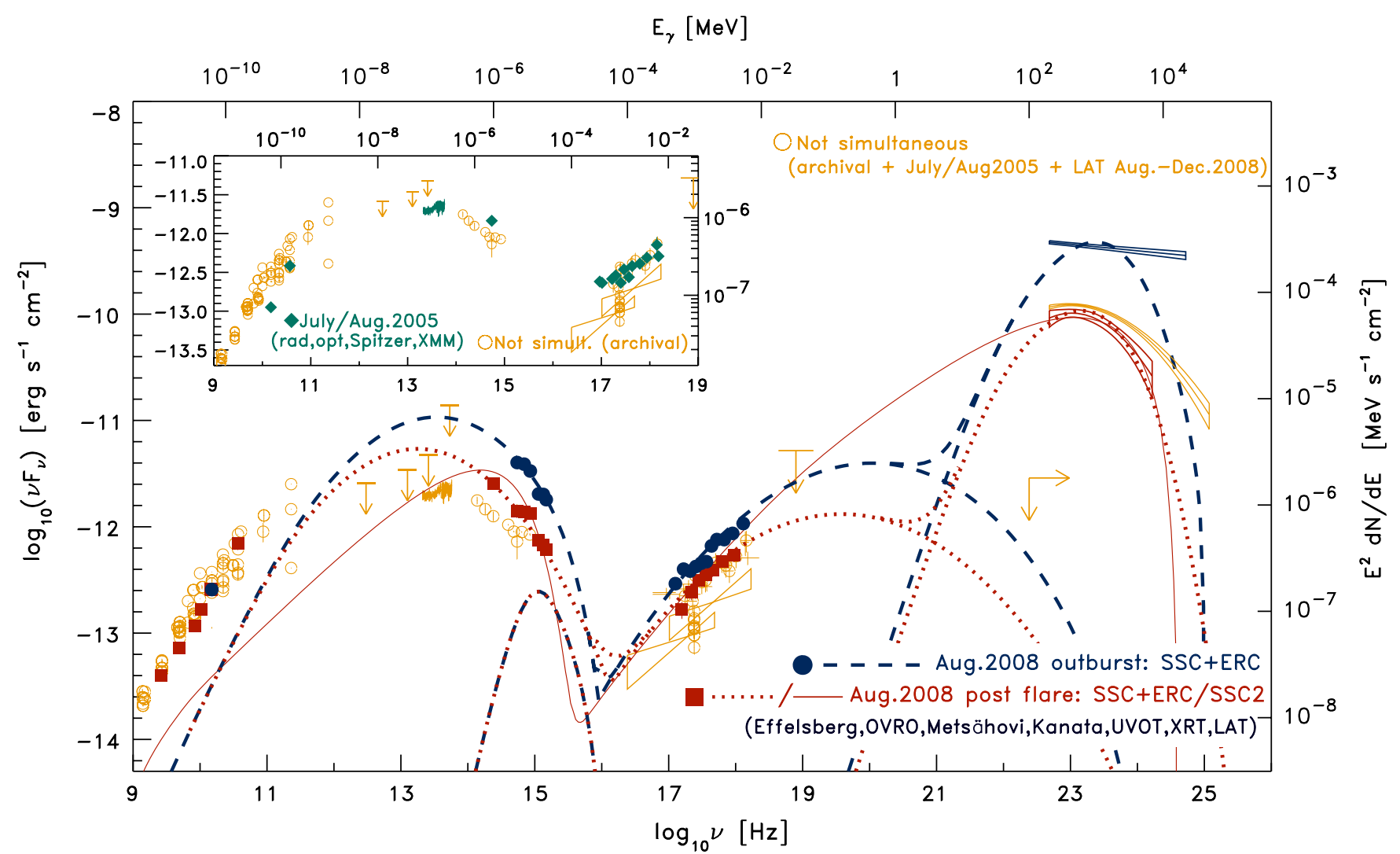

Figure 11. Major panel: overall radio-to-gamma-ray SED of PKS 1502+106 assembled with data from the two-week Fermi LAT multifrequency campaign of 2008 August. The two representative, time-averaged, states for this campaign (time intervals outlined in Figure 2), i.e., the high state (2008 August 5-10, DOY 2008: 218.95-224.0; blue filled circle symbol), and the post-flare (intermediate brightness) state (2008 August 11-22; DOY 2008: 224.0-235.42; red filled square symbol), are represented along with their SSC and ERC model attempts. Archival non-simultaneous data (including the 2005 July-August data, and the whole four-month cumulated LAT spectrum, open orange circle symbols and strip) are reported in the background for comparison. The high Compton dominance and gamma-ray bolometric luminosity reached during the outburst is evident. The SED of the outburst state is reported with a superposed one-zone "SSC+ERC" model fit attempt (where the SSC dominates the radio-to-X-ray SED and the ERC produces the gamma-ray component, blue dashed line), while the SED of the post-flare state is reported with two possible models superposed: the same SSC+ERC modeling (SSC radio-to-X-ray and ERC for gamma-ray band, red dotted line) and also a pure one-zone SCC attempt (labeled with "SSC2," a second type of stand-alone SSC model for the entire radio-to-gamma-ray SED, continuous red line). Inset panel: the 2005 July-August non-simultaneous SED (filled orange circles) assembled with the XMM-Newton EPIC-MOS data from 2005 July 17, the Spitzer IRS observation from 2005 August 13, radio flux data from Metsähovi and RATAN radio observatories, and optical data from the Catalina Sky Survey. Archival non-simultaneous data (open circles) are reported in background. This two-month averaged SED is consistent with a low or mildly active stage (suggested by the X-ray flux above $1 \times$ $10^{-12} \mathrm{erg} \mathrm{cm}^{-2} \mathrm{~s}^{-1}$ ) and can still be explained by a pure one-zone SSC model.

(A color version of this figure is available in the online journal.)

(Figure 4), appear well correlated, though the $V-J$ color was less pronounced than the multiband near-IR colors reported in Watanabe et al. (2004) and Figure 9, while the degree of optical polarization remained almost constant.

\subsection{Spectral Energy Distribution}

Variability is a powerful diagnostic to investigate blazar physics, but represents also a supplementary problem for the analysis of broadband SED, where model constraints are provided by simultaneous and well time-resolved multifrequency data. In the previous section, we indicated that dominant synchrotron and SSC (in-jet) mechanisms can explain the radioto-X-ray emission and correlations, whereas the origin of the high-power $\mathrm{MeV}-\mathrm{GeV}$ bolometric emission could be better constrained by external Comptonization of the radiation from the broad-line region (BLR), as invoked by Ghisellini et al. (2009), and found already in similar FSRQs of the EGRET era (e.g., Sokolov \& Marscher 2005; Sikora et al. 2008, 2009). The featureless mid-IR continuum observed by Spitzer-IRS in 2005 (Figure 9) supports the hypothesis of a prominent synchrotron emission by the jet, controlling the lower energy component. It could also reflect the lack of detectable ambient dust radiation, thereby supporting the idea that the ERC dissipation occurred within the BLR for this LAT outburst, in agreement with prescriptions of Sikora et al. (2002).

PKS 1502+106 might be considered a blazar peaked at the border of the $\mathrm{MeV}$ and $\mathrm{GeV}$ bands (a peak around 0.4 $0.5 \mathrm{GeV}$ is suggested by the curved model fit of the fourmonth spectrum; Section 2.4). In other words, PKS 1502+106 is likely at the border of the family of BLR-dissipated FSRQs and circum-nuclear ambient/torus dust-dissipated FSRQs, with an important SSC power output from radio-to-X-ray bands (Section 5.3), as depicted by the multiband correlations, the absence of hints for a bulk Compton feature produced by cold, adiabatically cooling electrons (as observed in PKS 1510-089; Kataoka et al. 2008; Abdo et al. 2010b), the lack of evidence for a blue bump.

The medium or high black hole mass of this blazar (likely in the range $0.5-1 \times 10^{9} M_{\odot}$, as calculated by D'Elia et al. 2003; Liu et al. 2006, respectively) could connote a BLR radiation field and IC dissipation moderately stronger than the magnetic energy density and the SSC luminosity in the gamma rays, as reported in Figure 11. The estimated accretion rate is $2 M_{\odot} \mathrm{yr}^{-1}$ (D'Elia et al. 2003). In this case, we have 
$L_{\mathrm{ERC}} / L_{\mathrm{syn}}=U_{\mathrm{BLR}}^{\prime} / U_{B} \simeq L_{\mathrm{BLR}} \mathcal{D}^{2} /\left(4 \pi c R_{\mathrm{BLR}}^{2} U_{B}\right)$, where $U_{\mathrm{BLR}}^{\prime}$ and $U_{B}$ are the electromagnetic energy density in the BLR and the magnetic field energy density in the jet blob, respectively. With a $10^{9} M_{\odot}$ mass, the Schwarzschild radius of the supermassive black hole is $R_{S}=2 G M / c^{2} \simeq 3 \times 10^{14} \mathrm{~cm}$. The bolometric luminosity of the BLR in PKS $1502+106$, evinced by the $\mathrm{Mg}$ II emission line profile, is $L_{\mathrm{BLR}}=3.7 \times 10^{45} \mathrm{erg} \mathrm{s}^{-1}$ (Liu et al. 2006), implying that the BLR is located at a radius $R_{\mathrm{BLR}}=$ $10^{17} \sqrt{L / 10^{45}} \simeq 2 \times 10^{17} \mathrm{~cm}$. We can expect relatively smaller magnetic fields in the dissipation region and an ERC, externaljet process that is the dominant cooling mechanism when the source is in a high $\gamma$-ray state, as suggested in Section 5.1. In fact, an SSC-only model would require a magnetic field in very sub-equipartition conditions, even if only the BLR fraction lying within the varying region's beaming cone could contribute to gamma rays. The simultaneous SED corresponding to the outburst state reported in Figure 11 corroborates the possible needs for an ERC contribution, showing a large $\left(L_{\gamma} / L_{\text {opt }} \sim 100\right)$ gamma-ray dominance over the synchrotron component. The SSC+ERC model can be considered in some way in agreement with the redshift value of this blazar, if we assume the FSRQs $\rightarrow$ BL Lac objects cosmological evolutionary scenario with dimming jet power, possibly related to star formation rate or the far-IR/submillimeter luminosity density (Dermer 2007).

In Figure 11, we report the data of the two SED states (the "outburst state" of 2008 August 5-10 (blue points) and the "post-flare" state (red points), characterized by an intermediate luminosity, of 2008 August 11-22 (both intervals are outlined in Figure 2), in conjunction with the unpublished archival and literature data collected for comparison. Above the two simultaneous SEDs of 2008 August, SSC and ERC "strawman" models are reported for each state (SSC+ERC modeling, blue lines for the outburst period, and SSC+ERC plus an SSC-standalone modeling, red lines, both for the post-flare period).

The pure SSC stand-alone model for the post-flare state (indicated in Figure 11 as a version 2 of the first-order SSC modeling with the label "SSC2," continuous red line, spanning from radio to gamma-ray bands) implements the temporal evolution of the synchrotron and SSC spectral components in a single flaring blob within the jet, where a population of accelerated electrons having a power law with exponential cutoff distribution $\left(d N / d E \propto E^{-p} e^{-\left(E / E_{\max }\right)}\right)$, is instantly injected (for more details on the analytical and numerical model see, e.g., Ciprini 2008). This form for $d N / d E$ is plausible in the presence of time-dependent acceleration or radiative-loss limits (Webb et al. 1984; Drury 1991). The post-flare (lower) state is reproduced by setting the electron energy index $p=1.77$, minimal and maximal electron Lorentz factors $\gamma_{\min }=200$, $\gamma_{\max }=8 \times 10^{4}$, compactness injected $\ell_{\mathrm{inj}}=L_{\mathrm{inj}} \sigma_{T} /\left(R m_{e} c^{3}\right)=$ $10^{-3}$, radius of the emitting region in the comoving frame $R=6.5 \times 10^{17} \mathrm{~cm}$, a bulk Doppler factor $\mathcal{D}=8$ and magnetic field intensity $B=0.024 \mathrm{G}$. If the X-ray spectrum is produced by electrons that cool on timescales longer than the light crossing time, $R / c$, the X-ray spectral index would be $\alpha_{X}=(p-1) / 2 \simeq 0.4$, a value quite similar to the averaged value $\left(\alpha_{X}=0.45 \pm 0.03\right)$ measured by Swift XRT for the post-flare interval (bottom segment of Table 2). The rather large size of the emitting region $(R \simeq 0.2 \mathrm{pc}$ ) and the very sub-equipartition magnetic field and reduced $\mathcal{D}$ suggested by this "SSC2" (stand-alone and first-order radio-to-gamma-ray SSC) model attempt indicate that even for this lower brightness state, an ERC contribution can be reasonable. Furthermore, the value of the optical-UV spectral index for this post-flare state $\left(\alpha_{\mathrm{UVOT}}=1.9 \pm 0.3\right)$ is softer than the averaged index (3/2) expected (Chiang \& Böttcher 2002), when a totally SSCdominated loss is considered. The huge Compton dominance of the outburst state might preclude a first-order SSC-standalone model attempt for such an SED state. On the other hand, for high-energy $\gamma$-ray loud blazars like PKS 1502+106 superquadratic variations produced by higher order scatters in SSC are predicted by other models (Georganopoulos et al. 2006; Perlman et al. 2008) and Section 5.1, in fact in luminous blazars, the optical depth to Compton scattering (related to particle density) increases and second and higher order scatters become more important. In more Compton-dominated objects, these scattering reactions can dominate the energy output from the SSC process, and can produce superquadratic behaviors during big flares.

As mentioned above, an "SSC plus ERC" model description, joining an SSC radio-to-X-ray component and an ERC gamma-ray component, to both the post-flare and outburst states (Figure 11, dashed blue and red dotted lines) is plausible as well for these SEDs. The description of an initial version of this composite modeling, implementing the prescriptions of Sikora et al. (1994); Dermer \& Schlickeiser (2002), can be found, e.g., in Tramacere \& Tosti (2003). The low state is described with the following "SSC+ERC" parameters $B=0.5 \mathrm{G}$, blob size $R=7.9 \times 10^{16} \mathrm{~cm}, \mathcal{D}=20$, an LP electron injection function between $\gamma_{\min }=100$ and $\gamma_{\max }=3 \times 10^{4}$, $L_{\text {disk }}=1.1 \times 10^{46}, \tau_{\mathrm{BLR}}=0.1, T_{\text {disk }}=1.5 \times 10^{5} \mathrm{~K}$ and distance from the disk of $10^{18} \mathrm{~cm}$. The outburst state is described with the following "SSC+ERC" parameters $B=0.5 \mathrm{G}$, blob size $R=6.3 \times 10^{16} \mathrm{~cm}, \mathcal{D}=24$, an LP electron injection function between $\gamma_{\min }=100$ and $\gamma_{\max }=3 \times 10^{4}, L_{\text {disk }}=1.1 \times 10^{46}$, $\tau_{\mathrm{BLR}}=0.1, T_{\text {disk }}=1.5 \times 10^{5} \mathrm{~K}$ and distance from the disk of $7 \times 10^{17} \mathrm{~cm}$.

In Figure 11, the LAT-averaged spectrum $(E>200 \mathrm{MeV})$ of the entire 2008 August-December period considered (characterized by high statistics and consistent with an intrinsically curved shape described by an LP model) is reported as well (the strip in the same orange color used for non-simultaneous data). LP curvature (Section 2.4) at high energy can be produced by several plausible models. For example, by radiative particle cooling and stochastic acceleration processes driven by magnetic turbulence (rather than systematic particle acceleration) acting near the shock front (Stawarz \& Petrosian 2008). If the energy where losses balance the acceleration rate if the acceleration time decreases more slowly than the loss time. The probability of energetic gain is lower when particle energy increases, because particles are confined by a magnetic field with a confinement efficiency decreasing for an increasing gyration radius. The integral energy distribution of the accelerated particles results in an LP law: $N(\gamma)=N_{0}\left(\gamma / \gamma_{0}\right)^{-p-1+r \log \left(\gamma / \gamma_{0}\right)}$, where $r$ is a curvature term (Landau et al. 1986; Fossati et al. 2000; Massaro et al. 2004; Perlman et al. 2005; Tramacere et al. 2007). Non-power-law radiation spectra could also originate in a nonlinear regime, such as in the presence of shock modifications, precursors, and when diffusion coefficients vary with particle momentum (Amato et al. 2008). Another explanation takes into account episodic particle acceleration that is applicable to high-energy flares with intrinsic spectral curvature (Perlman et al. 2005; Perlman \& Wilson 2005). The filling factor of the regions within which particles are accelerated is a function of both position and energy. If the light-crossing time of the emission region or the integration time of observations is greater than the characteristic particle acceleration time, we 
effectively observe an electron distribution which is the product of a power law multiplied by a logarithmic term producing a spectral curvature.

Another contribution to such deviations might also be the averaging over long periods of time, hence combining variability effects, resulting in a cumulation of photons from different activity "flavors" (different source brightness and spectral hardness) being included in the evaluation of the spectrum. In the case of PKS $1502+106$, the same spectral curved shape was also preferred for the much shorter, post-flare, interval, while the whole period includes many photons and covers a wider energy range (photons observed from the source have energies up to $15.8 \mathrm{GeV}$ ). PKS 1502+106, and 3C 454.3 (Abdo et al. 2009a) for the first time show this departure from a simple power law, while EGRET observed only simple power laws, probably due to its lower high-energy observational limit. The maximum peak observed in the LP models of PKS 1502+106 (in the $v F_{v}$ representation) are around energies of about $390 \mathrm{MeV}$ for the whole period and $480 \mathrm{MeV}$ for the post-flare period. These peaks are consistent with a sub-GeV FSRQ blazar class.

From Section 5.1, we remember that the inferred, apparent and isotropic, monochromatic luminosity at $E_{0}=100 \mathrm{MeV}$ during the outburst phase (DOY 2008: 218.95-224.0) is $L_{E>100} \simeq$ $1.1 \times 10^{49} \mathrm{erg} \mathrm{s}^{-1}$, and the bolometric luminosity is expected to be even higher than this value, making PKS 1502+106 one of the most powerful and luminous high-energy blazars observed during the first year the Fermi LAT all-sky survey. On the other hand, the highest energy of photons detected from the source during the first four months of survey $(15.8 \mathrm{GeV}$, assuming a strict PSF size criterium) has marginal consequences for extragalactic background light (EBL) predictions. The optical depth for $\gamma \gamma \rightarrow e^{+} e^{-}$pair production of $16 \mathrm{GeV}$ photons propagating through the EBL from a redshift $z=1.839$ source to Earth approaches unity for rather high-density EBL models (e.g., Stecker et al. 2006, $\left.\tau_{\gamma \gamma}(z=1.839, E=16 \mathrm{GeV}) \sim 1.0-1.3\right)$, while most low-density EBL models predict rather small interaction probabilities at such energies. An in-depth exploration of this finding will be presented elsewhere (A. A. Abdo et al. 2010, in preparation).

\section{FINAL REMARKS}

This was the first time that PKS $1502+106$, a distant radio- and $\mathrm{X}$-ray-selected FSRQ, has been announced to have observable high-energy gamma-ray emission above $100 \mathrm{MeV}$. Fermi LAT as basically a sort of all-space, -time, and -energy monitor allowed an excellent spatial localization of this new $\gamma$-ray source (Section 2.2 and Figure 1, with a firm identification thanks to the optical- $\gamma$-ray match of the outburst and, remarkably, also of a second flare). It allowed detailed analysis of the energy spectrum (Section 2.4 and Figure 11, demonstrating the possibility for an intrinsic spectral curvature in $\gamma$ rays), and was able to provide regular, daily monitoring flux light curve (Section 2.3 and Figure 2). This has made possible the discovery of consistent $\gamma$-ray brightness and activity ignited by the big outburst over the following four months (pointing out a $1 / f^{1.3}$ variability behavior), the discovery of further minor and rapid flares, and the disclosure of the outburst's temporal shape. This type of "PSD-SED" monitoring performed by the LAT yielded advantage in developing the first unplanned Fermi multifrequency campaign, with a strategic 16 days of simultaneous Fermi-Swift monitoring.

PKS $1502+106$ is a powerful gamma-ray $\left(\sim 10^{49} \mathrm{erg} \mathrm{s}^{-1}\right.$ at $E>100 \mathrm{MeV}$ ) FSRQ that showed, especially during the fast-rising outburst, a dominant $\mathrm{MeV}-\mathrm{GeV}$ bolometric emission similar to other FSRQs of the EGRET era. Dissipation probably occurred within the BLR, and, assuming for a black hole mass of $\sim 10^{9} M_{\odot}$, the $\gamma$-ray emission was likely dominated by the ERC process. The SSC, in-jet, emission appears to dominate the observed SED from radio to X-rays bands. PKS 1502+106 might be considered an example of a sub-GeV peaked blazar, placed at the border of the BLR dissipated and the dusty torus/ambientradiation dissipated FSRQs classes. The level of correlation found among the $\gamma$-ray, X-ray, and optical-UV outburst and post-flare relaxing phases, support this idea. Opacity effects at $\mathrm{cm}$ and longer radio wavelengths, possible links between field ordering, jet-axis alignment, superluminal radio knots, and $\mathrm{MeV}-\mathrm{GeV}$ outburst, are also depicted by our results.

In conclusion, the Fermi LAT performance in blazar science (as a stand-alone observatory, or leading instrument for multifrequency campaigns), and the synergy between Fermi and Swift in particular, is evidenced by this work on PKS 1502+106. By itself, this blazar is emerging as a major, luminous, energetic, and $\gamma$-ray variable source, with promising diagnostic and discovery potential in emission modeling, in spectral and temporal variability studies, and in understanding the radio-gamma-ray connection.

This research is based on observations obtained with the Fermi Gamma-ray Space Telescope. The Fermi LAT Collaboration acknowledges generous ongoing support from a number of agencies and institutes that have supported both the development and the operation of the LAT as well as scientific data analysis. These include the National Aeronautics and Space Administration and the Department of Energy in the United States, the Commissariat à l'Energie Atomique and the Centre National de la Recherche Scientifique/Institut National de Physique Nucléaire et de Physique des Particules in France, the Agenzia Spaziale Italiana (ASI) and the Istituto Nazionale di Fisica Nucleare (INFN) in Italy, the Ministry of Education, Culture, Sports, Science and Technology (MEXT), High Energy Accelerator Research Organization (KEK), and Japan Aerospace Exploration Agency (JAXA) in Japan, and the K. A. Wallenberg Foundation, the Swedish Research Council, and the Swedish National Space Board in Sweden. Additional support for science analysis during the operations phase is gratefully acknowledged from the Istituto Nazionale di Astrofisica (INAF) in Italy and the Centre National d'Études Spatiales in France. S.C. acknowledges funding by grant ASI-INAF n.I/047/8/0 related to Fermi onorbit activities. This research has made use of the NASA/IPAC NED database (JPL CalTech and NASA, USA), the HEASARC database (LHEA NASA/GSFC and SAO, USA), the Smithsonian/NASA's ADS bibliographic databases, and the SIMBAD database (CDS, Strasbourg, France). This work includes observations obtained with the NASA Swift gamma-ray burst Explorer. This work includes observations obtained with the Spitzer Space Telescope (operated by the Jet Propulsion Laboratory, California Institute of Technology under a contract with NASA). This work includes observations obtained with XMM-Newton, an ESA science mission with instruments and contributions directly funded by ESA Member States and NASA. This work has made use of observations obtained with the Owens Valley Radio Observatory. The monitoring program at the Owens Valley Radio Observatory is supported by NASA award No. NNX08AW31G, and NSF award No. AST-0808050. This research has made use of observations from the MOJAVE database that is maintained by the MOJAVE team. The MOJAVE project 
is supported under National Science Foundation grant 0807860AST and NASA-Fermi grant NNX08AV67G. The National Radio Astronomy Observatory (NRAO VLBA) is a facility of the National Science Foundation operated under cooperative agreement by Associated Universities, Inc. This research has made use of observations obtained with the $100 \mathrm{~m}$ telescope of the MPIfR (Max-Planck-Institut für Radioastronomie) at Effelsberg, Germany. This research has made use of observations from the RATAN-600 that is partly supported by the Russian Foundation for Basic Research (projects 01-02-16812, 05-0217377, and 08-02-00545). This work has made use of observations obtained with the $14 \mathrm{~m}$ Metsähovi Radio Observatory, a separate research institute of the Helsinki University of Technology. The Metsähovi team acknowledges the support from the Academy of Finland. This work has made use of observations obtained with the TRISPEC instrument on the Kanata telescope that is operated by Hiroshima University, Japan. Y.Y.K. is a Research Fellow of the Alexander von Humboldt Foundation. The LAT team and multifrequency collaboration extend thanks to the anonymous referee who made very useful comments.

Facilities: Fermi, Swift, VLBF, XMM, Spitzer, INTEGRAL

\section{REFERENCES}

Abdo, A. A., et al. 2009a, ApJ, 699, 817

Abdo, A. A., et al. 2009b, ApJ, 700, 597

Abdo, A. A., et al. 2009c, ApJS, 183, 46

Abdo, A. A., et al. 2009d, Astropart. Phys., 32, 193

Abdo, A. A., et al. 2010a, ApJ, submitted (AGN variability)

Abdo, A. A., et al. 2010b, ApJ, submitted (PKS 1510-089)

Aharonian, F., et al. 2007, ApJ, 664, L71

Akiyama, M., Ueda, Y., Ohta, K., Takahashi, T., \& Yamada, T. 2003, ApJS, 148, 275

Aller, M. F., Aller, H. D., Hughes, P. A., \& Latimer, G. E. 1999, ApJ, 512, 601 Amato, E., Blasi, P., \& Gabici, S. 2008, MNRAS, 385, 1946

An, T., Hong, X. Y., Venturi, T., Jiang, D. R., \& Wang, W. H. 2004, A\&A, 421, 839

Angelakis, E., Fuhrmann, L., Marchili, N., Krichbaum, T. P., \& Zensus, J. A. 2008, Mem. SAIt, 79, 1042

Argue, A. N., \& Sullivan, C. 1980, Observatory, 100, 152

Atwood, W. B., et al. 2007, Astropart. Phys., 28, 422

Atwood, W. B., et al. 2009, ApJ, 697, 1071

Baars, J. W. M., Genzel, R., Pauliny-Toth, I. I. K., \& Witzel, A. 1977, A\&A, 61,99

Begelman, M. C., Blandford, R. D., \& Rees, M. J. 1984, Rev. Mod. Phys., 56, 255

Bellazzini, R., et al. 2002, Nucl. Phys. B: Proc. Suppl., 113, 303

Bertin, E., \& Arnouts, S. 1996, A\&AS, 117, 393

Blake, G. M. 1970, Astrophys. Lett., 6, 201

Böttcher, M., \& Chiang, J. 2002, ApJ, 581, 127

Burbidge, E. M., \& Strittmatter, P. A. 1972, ApJ, 174, L57

Burnett, T. H. 2007, in AIP Conf. Proc. 921, The First GLAST Symposium, ed. S. Ritz, P. Michelson, \& C. A. Meegan (Melville, NY: AIP), 530

Camilo, F., et al. 2009, ApJ, 705, 1

Casandjian, J.-M., \& Grenier, I. A. 2008, A\&A, 489, 849

Cecchi, C., et al. 2007, in AIP Conf. Proc. 921, The First GLAST Symposium, ed. S. Ritz, P. Michelson, \& C. A. Meegan (Melville, NY: AIP), 540

Chiaberge, M., \& Ghisellini, G. 1999, MNRAS, 306, 551

Chiang, J., \& Böttcher, M. 2002, ApJ, 564, 92

Chiang, J., Carson, J., \& Focke, W. 2007, in AIP Conf. Proc. 921, The First GLAST Symposium, ed. S. Ritz, P. Michelson, \& C. A. Meegan (Melville, NY: AIP), 544

Chiang, J., Digel, S., Silva, E. D. C. E., \& Reimer, O. 2006, BAAS, 38, 382

Ciaramella, A., et al. 2004, A\&A, 419, 485

Ciprini, S. 2008, Proc. of Blazar Variability Across the Electromagnetic Spectrum, PoS(BLAZARS2008), n.073

Ciprini, S., et al. 2007a, in AIP Conf. Proc. 921, The First GLAST Symposium, ed. S. Ritz, P. Michelson, \& C. A. Meegan (Melville, NY: AIP), 546

Ciprini, S., et al. 2007b, A\&A, 467, 465

Cooper, N. J., Lister, M. L., \& Kochanczyk, M. D. 2007, ApJS, 171, 376

Crowther, J. H., \& Clarke, R. W. 1966, MNRAS, 132, 405

Damiani, F., Maggio, A., Micela, G., \& Sciortino, S. 1997, ApJ, 483, 350
D'Arcangelo, F. D., et al. 2007, ApJ, 659, L107

Day, G. A., Shimmins, A. J., Ekers, R. D., \& Cole, D. J. 1966, Aust. J. Phys., 19,35

D'Elia, V., Padovani, P., \& Landt, H. 2003, MNRAS, 339, 1081

Dermer, C. D. 2007, ApJ, 659, 958

Dermer, C. D., \& Schlickeiser, R. 2002, ApJ, 575, 667

Drury, L. O. 1991, MNRAS, 251, 340

Fichtel, C. E., et al. 1994, ApJS, 94, 551

Fitch, L. T., Dixon, R. S., \& Kraus, J. D. 1969, AJ, 74, 612

Fossati, G., et al. 2000, ApJ, 541, 166

Fuhrmann, L., Zensus, J. A., Krichbaum, T. P., Angelakis, E., \& Readhead, A. C. S. 2007, in AIP Conf. Proc. 921, The First GLAST Symposium, ed. S. Ritz, P. Michelson, \& C. A. Meegan (Melville, NY: AIP), 249

Fuhrmann, L., et al. 2008, A\&A, 490, 1019

Gehrels, N., et al. 2004, ApJ, 611, 1005

Georganopoulos, M., \& Marscher, A. P. 1998, ApJ, 506, L11

Georganopoulos, M., Perlman, E. S., Kazanas, D., \& Wingert, B. 2006, AIP Conf. Proc., 350, 178

George, I. M., Nandra, K., Turner, T. J., \& Celotti, A. 1994, ApJ, 436, L59

Ghisellini, G., \& Tavecchio, F. 2009, MNRAS, 397, 985

Ghisellini, G., Tavecchio, F., \& Ghirlanda, G. 2009, MNRAS, 399, 2041

Graff, P. B., Georganopoulos, M., Perlman, E. S., \& Kazanas, D. 2008, ApJ, 689,68

Hartman, R. C., et al. 1999, ApJS, 123, 79

Hartman, R. C., et al. 2001, ApJ, 553, 683

Healey, S. E., et al. 2008, ApJS, 175, 97

Houck, J. R., et al. 2004, ApJS, 154, 18

Hovatta, T., Tornikoski, M., Lainela, M., Lehto, H. J., Valtaoja, E., Torniainen, I., Aller, M. F., \& Aller, H. D. 2007, A\&A, 469, 899

Hovatta, T., Valtaoja, E., Tornikoski, M., \& Lähteenmäki, A. 2009, A\&A, 494, 527

Hughes, P. A., Aller, H. D., \& Aller, M. F. 1992, ApJ, 396, 469

Inoue, S., \& Takahara, F. 1996, ApJ, 463, 555

Jorstad, S., et al. 2007, AJ, 134, 799

Kataoka, J., et al. 2008, ApJ, 672, 787

Kellermann, K. I., et al. 2004, ApJ, 609, 539

Kirk, J. G., Rieger, F. M., \& Mastichiadis, A. 1998, A\&A, 333, 452

Komatsu, E., et al. 2009, ApJS, 180, 330

Korolkov, D. V., \& Parijskij, Yu. N. 1979, Sky Telesc., 57, 324

Kovalev, Y. Y., Nizhelsky, N. A., Kovalev, Yu. A., Berlin, A. B., Zhekanis, G. V., Mingaliev, M. G., \& Bogdantsov, A. V. 1999, A\&AS, 139, 545

Kovalev, Y. Y., et al. 2005, AJ, 130, 2473

Kovalev, Y. Y., et al. 2009, ApJ, 696, L17

Landau, R., et al. 1986, ApJ, 308, 78

Leipski, C., et al. 2009, ApJ, 701, 891

Lister, M. L., Aller, H. D., \& Aller, M. F. 2009a, AJ, 137, 3718

Lister, M. L., \& Homan, D. C. 2005, AJ, 130, 1389

Lister, M. L., Homan, D. C., Kadler, M., Kellermann, K. I., Kovalev, Y. Y., Ros, E., Savolainen, T., \& Zensus, J. A. 2009b, ApJ, 696, L22

Liu, Y., Jiang, D. R., \& Gu, M. F. 2006, ApJ, 637, 669

López-Caniego, M., et al. 2007, ApJS, 170, 108

Lott, B., et al. 2007, in AIP Conf. Proc. 921, The First GLAST Symposium, ed. S. Ritz, P. Michelson, \& C. A. Meegan (Melville, NY: AIP), 347

Madejski, G., et al. 1996, ApJ, 459, 156

Marcucci, F., Cecchi, C., \& Tosti, G. 2004, Frascati Phys. Ser., 37, 285

Marscher, A. P., et al. 2008, Nature, 452, 966

Massaro, E., Perri, M., Giommi, P., \& Nesci, R. 2004, A\&A, 413, 489

Massaro, E., et al. 2009, A\&A, 495, 691

Mattox, J. R., et al. 1993, ApJ, 410, 609

Mattox, J. R., et al. 1996, ApJ, 461, 396

McEnery, J. 2006, in ASP Conf. Ser. 350, Blazar Variability Workshop II: Entering the GLAST Era, ed. H. R. Miller, K. Marshall, J. R. Webb, \& M. F. Aller (San Francisco, CA: ASP), 229

Michelson, P. F. 2007, in AIP Conf. Proc. 921, The First GLAST Symposium, ed. S. Ritz, P. Michelson, \& C. A. Meegan (Melville, NY: AIP), 8

Moskalenko, I. V., Jones, F. C., Mashnik, S. G., Ptuskin, V. S., \& Strong, A. W. 2003, Proc. 28th ICRC (Trukuba), 4, 1925

Neugebauer, G., Miley, G. K., Soifer, B. T., \& Clegg, P. E. 1986, ApJ, 308, 815

Nolan, P. L., Tompkins, W. F., Grenier, I. A., \& Michelson, P. F. 2003, ApJ, 597, 615

Pei, Y. C. 1992, ApJ, 395, 130

Perlman, E., Addison, B., Georganopoulos, M., Wingert, B., \& Graff, P. 2008, Proc. of Blazar Variability Across the Electromagnetic Spectrum, PoS(BLAZARS2008), n.009

Perlman, E. S., \& Wilson, A. S. 2005, ApJ, 627, 140

Perlman, E. S., et al. 2005, ApJ, 625, 727

Petrucci, P. O., et al. 2002, A\&A, 388, L5 
Poole, T. S., et al. 2008, MNRAS, 383, 627

Readhead, A. C. S., Lawrence, C. R., Myers, S. T., Sargent, W. L. W., Hardebeck, H. E., \& Moffet, A. T. 1989, ApJ, 346, 566

Ritz, S. 2007, in AIP Conf. Proc. 921, The First GLAST Symposium, ed. S. Ritz, P. Michelson, \& C. A. Meegan (Melville, NY: AIP), 3

Schlegel, D. J., Finkbeiner, D. P., \& Davis, M. 1998, ApJS, 500, 525

Sikora, M., Begelman, M. C., \& Rees, M. J. 1994, ApJ, 421, 153

Sikora, M., Błażejowski, M., Begelman, M. C., \& Moderski, R. 2001, ApJ, 554, 1

Sikora, M., Błażejowski, M., Moderski, R., \& Madejski, G. M. 2002, ApJ, 577, 78

Sikora, M., Moderski, R., \& Madejski, G. M. 2008, ApJ, 675, 71

Sikora, M., Stawarz, Ł., Moderski, R., Nalewajko, K., \& Madejski, G. M. 2009, ApJ, 704, 38

Smith, H. E., Burbidge, E. M., Baldwin, J. A., Tohline, J. E., Wampler, E. J., Hazard, C., \& Murdoch, H. S. 1977, ApJ, 215, 427

Sokolov, A., \& Marscher, A. P. 2005, ApJ, 629, 52

Sokolov, A., Marscher, A. P., \& McHardy, I. M. 2004, ApJ, 613, 725

Sowards-Emmerd, D., Romani, R. W., \& Michelson, P. F. 2003, ApJ, 590, 109

Sowards-Emmerd, D., et al. 2005, ApJ, 626, 95

Starck, J.-L., \& Pierre, M. 1998, A\&AS, 128, 397

Stawarz, Ł., \& Petrosian, V. 2008, ApJ, 681, 1725

Stecker, F. W., Malkan, M. A., \& Scully, S. T. 2006, ApJ, 648, 774

Strüder, L., et al. 2001, A\&A, 365, L18
Teräsranta, H., Wiren, S., Koivisto, P., Saarinen, V., \& Hovatta, T. 2005, A\&A, 440,409

Teräsranta, H., et al. 1998, A\&AS, 132, 305

Thompson, D. J. 2006, in ASP Conf. Ser. 350, Blazar Variability Workshop II: Entering the GLAST Era, ed. H. R. Miller, K. Marshall, J. R. Webb, \& M. F. Aller (San Francisco, CA: ASP), 113

Thompson, D. J. 2007, in AIP Conf. Proc. 921, The First GLAST Symposium, ed. S. Ritz, P. Michelson, \& C. A. Meegan (Melville, NY: AIP), 86

Thompson, D. J., et al. 1996, ApJS, 107, 227

Tosti, G. 2007, in AIP Conf. Proc. 921, The First GLAST Symposium, ed. S. Ritz, P. Michelson, \& C. A. Meegan (Melville, NY: AIP), 255

Tramacere, A., Massaro, F., \& Cavaliere, A. 2007, A\&A, 466, 521

Tramacere, A., \& Tosti, G. 2003, New Astron. Rev., 47, 697

Uemura, M., et al. 2008, Proc. of Blazar Variability Across the Electromagnetic Spectrum, PoS(BLAZARS2008), n.070

Watanabe, C., Ohta, K., Akiyama, M., \& Ueda, Y. 2004, ApJ, 610, 128

Watanabe, M., et al. 2005, PASP, 117, 870

Webb, G. M., Drury, L. O., \& Biermann, P. 1984, A\&A, 137, 185

Wehrle, A., et al. 1998, ApJ, 497, 178

Wilkes, B. J., Wright, A. E., Jauncey, D. L., \& Peterson, B. A. 1983, PASAu, 5, 2

Williams, P. J. S., Kenderdine, S., \& Baldwin, J. E. 1967, MmRAS, 70, 53

Wright, A. E., Peterson, B. A., Jauncey, D. L., \& Condon, J. J. 1979, ApJ, 229, 73 